\title{
Dynamical critical scaling of electric field fluctuations in the greater cusp and magnetotail implied by HF radar observations of F-region Doppler velocity
}

\author{
M. L. Parkinson \\ Department of Physics, La Trobe University, Bundoora Campus, Victoria 3086, Australia \\ Received: 6 July 2005 - Revised: 23 January 2006 - Accepted: 27 January 2006 - Published: 23 March 2006
}

\begin{abstract}
Akasofu's solar wind $\varepsilon$ parameter describes the coupling of solar wind energy to the magnetosphere and ionosphere. Analysis of fluctuations in $\varepsilon$ using model independent scaling techniques including the peaks of probability density functions (PDFs) and generalised structure function (GSF) analysis show the fluctuations were self-affine (mono-fractal, single exponent scaling) over 9 octaves of time scale from $\sim 46 \mathrm{~s}$ to $\sim 9.1 \mathrm{~h}$. However, the peak scaling exponent $\alpha_{0}$ was a function of the fluctuation bin size, so caution is required when comparing the exponents for different data sets sampled in different ways. The same generic scaling techniques revealed the organisation and functional form of concurrent fluctuations in azimuthal magnetospheric electric fields implied by SuperDARN HF radar measurements of line-of-sight Doppler velocity, $v_{\mathrm{LOS}}$, made in the high-latitude austral ionosphere. The PDFs of $v_{\text {LOS }}$ fluctuation were calculated for time scales between $1 \mathrm{~min}$ and $256 \mathrm{~min}$, and were sorted into noon sector results obtained with the Halley radar, and midnight sector results obtained with the TIGER radar. The PDFs were further sorted according to the orientation of the interplanetary magnetic field, as well as ionospheric regions of high and low Doppler spectral width. High spectral widths tend to occur at higher latitude, mostly on open field lines but also on closed field lines just equatorward of the open-closed boundary, whereas low spectral widths are concentrated on closed field lines deeper inside the magnetosphere. The $v_{\text {LOS }}$ fluctuations were most self-affine (i.e. like the solar wind $\varepsilon$ parameter) on the high spectral width field lines in the noon sector ionosphere (i.e. the greater cusp), but suggested multi-fractal behaviour on closed field lines in the midnight sector (i.e. the central plasma sheet). Long tails in the PDFs imply that "microbursts" in ionospheric convection occur far more frequently, especially on open field lines, than can be captured
\end{abstract}

Correspondence to: M. L. Parkinson

(m.parkinson@latrobe.edu.au) using the effective Nyquist frequency and volume resolution of SuperDARN radars.

Keywords. Ionosphere (Electric fields and currents) - Magnetospheric physics (Plasma sheet) - Space plasma physics (Nonlinear phenomena)

\section{Introduction}

Some complex physical systems can establish a scale-free state known as self-organised criticality (SOC) (Bak et al., 1987; Frette et al., 1996). These systems exhibit burstiness, intermittency, and self-similarity over many orders of spatial and temporal scale. Familiar examples of terrestrial systems exhibiting SOC may include earthquakes, the accumulation of snow on mountain tops, and water drops on a pane of glass (Jensen, 1998). All undergo potentially catastrophic energy release via localised or system-wide avalanches, a defining feature of SOC. The intermittency of avalanches means the probability of major, sometimes life-threatening energy release is far greater than predicted by simple Gaussian statistics. For example, nivologists aim to incorporate the powerlaw behaviour of self-organised systems into routine predictions of avalanche risk for the European Alps (Palmer, 2003). This is an example of the way in which complexity science might contribute to the preservation of human life.

Solar flares (Lu, 1995), the solar wind (Freeman et al., 2000; Hnat et al., 2003a), and the magnetosphere (Klimas et al., 2000; Baker et al., 1999; Consolini and Chang, 2001), are good examples of astrophysical systems exhibiting the intermittency characteristic of SOC. However, some aspects of their statistical behaviour may also be characteristic of other kinds of non-linear dynamics (Sornette, 2000). Fluctuations within systems exhibiting the same statistical behaviour may be governed by the same set of non-linear equations, irrespective of the "micro" physics governing them.

Published by Copernicus GmbH on behalf of the European Geosciences Union. 
For example, Hnat et al. (2003b) modeled the probability density functions (PDFs) of solar wind fluctuations using a generalised Fokker-Planck equation (Sornette, 2000) and a Castaing model (Castaing et al., 1990). Statistical physics approaches like these provide constraints on first principle physics models, such as magneto-hydrodynamic (MHD) models incorporating kinetic effects, which must ultimately describe the same kind of non-linear behaviour. Studies like these are also important because they will contribute to the prediction of the risk of space weather events such as solar flares and substorms.

The extent to which fluctuations of currents and electric fields within the magnetosphere and ionosphere are driven by, or independent of, corresponding fluctuations in the solar wind is still an open question (Freeman et al., 2000; Uritsky et al., 2001). It is well known that electric field fluctuations within the greater cusp are strongly coupled to the fluctuations in the solar-wind via magnetic reconnection (Pinnock et al., 1993). In this paper the greater cusp refers to the ionospheric projection of the dayside low latitude boundary layer (LLBL), cusp, cleft, and adjacent mantle.

However, there is nearly always a background component of electric potential and current flow that is not directly controlled by the interplanetary magnetic field (IMF) (Kelley, 1989). In fact, spatial fluctuation of electric fields in the high-latitude ionosphere are strongest during IMF northward conditions, when coupling to the solar wind is weakest (Golovchanskaya et al., 2002). This was attributed to the interchange instability acting over extended regions of closed magnetic flux when $B_{z}$ is northward.

There might be characteristic variations in the electrodynamic fluctuations, and the dynamical equations governing them, for different phases of the sunspot cycle, different states of the IMF, and in different magnetospheric regions. For example, it is well known that changes in the IMF rapidly couple to the dayside ionosphere and magnetosphere, and probably the nightside ionosphere and magnetotail, at least for some events (e.g., see Ruohoniemi et al., 2002). However, the spectacular consequence of these IMF changes, the onset of magnetospheric substorms and the development of a distinct current systems which include the substorm current wedge, are often delayed. Certainly, there must be differences between instabilities acting in the cusp and magnetotail.

A recent study by Hnat et al. (2005) suggests that fluctuations in auroral electrojet indices are partly driven by the solar wind, yet partly independent, hence suggesting selforganised behaviour in the magnetotail. Although some substorms are triggered by IMF northward turnings, and the solar wind may be the ultimate source of energy, it is well known that substorm evolution is not necessarily directly driven by solar wind variability. Substorm evolution can also be dominated by reconnection of closed magnetic flux in the central plasma sheet (CPS) (e.g. Lester et al., 2005 ${ }^{1}$ ). Unlike the fluctuations in solar wind parameters which are monofractal, electric field fluctuations associated with current disruption in the CPS may be multi-fractal at time scales less than the substorm cycle (e.g. Lui, 2001; Vörös et al., 2003).

The aim of this paper is to identify the characteristics of magnetospheric electric field fluctuations implied by Super Dual Auroral Radar Network (SuperDARN) observations of line-of-sight Doppler velocity, $v_{\mathrm{LOS}}$, in the noon and midnight sectors of the high-latitude ionosphere. Magnetospheric electric fields map to ionospheric electric fields, except at short scales in regions of strong Pedersen conductance and field-aligned potential drops (Weimer et al., 1985). Hence the characteristics of electric field fluctuations may differ depending on whether they occur within regions of low Pedersen conductance which tend to occur in the weakly illuminated polar cap ionosphere (open field lines), or within regions of high Pedersen conductance which tend to occur in nightside auroral regions (closed field lines) (see Parkinson et al., 2004).

Here we analyse Advanced Composition Explorer (ACE) spacecraft measurements of the solar wind and SuperDARN HF radar measurements of $v_{\text {LOS }}$ for the year 2000. First we characterise fluctuations in the solar wind $\varepsilon$ parameter by calculating the probability density functions (PDFs) and statistical moments of fluctuations in $\varepsilon$ on various time scales. Next we characterise concurrent fluctuations in $v_{\text {LOS }}$ by calculating PDFs sorted according to IMF orientation, magnetic local time (MLT), and approximate regions of open and closed magnetic flux. The different sets of PDFs are re-scaled using the generic and model independent techniques described by Hnat et al. (2002b, 2003a, 2005). The extent to which the PDFs collapse to a common curve reveals whether they are self-affine (mono-fractal, single exponent scaling) or possibly multi-fractal (e.g. Lui, 2001; Vörös et al., 2003). These results provide further insights into the dynamics governing the behaviour of the coupled ionosphere-magnetosphere system.

\section{Scaling of the solar wind $\varepsilon$ parameter}

\subsection{Instruments and data set}

The ACE spacecraft was located at about 235 Re upstream of the Earth, and provided nearly continuous measurements of solar wind conditions throughout the year 2000. We obtained measurements of the solar wind speed $v_{S W}$ (McComas et al., 1998) measured in geocentric solar magnetospheric (GSM) co-ordinates at 64-s time resolution, and the three IMF components $\left(B_{z}, B_{y}, B_{z}\right)$ (Smith et al., 1998) measured

\footnotetext{
${ }^{1}$ Lester, M., Parkinson, M. L., Wild, J. A., et al.: Simultaneous observations of ionospheric flow and tail reconnection signatures during the substorm expansion phase, Ann. Geophys., submitted, 2005.
} 


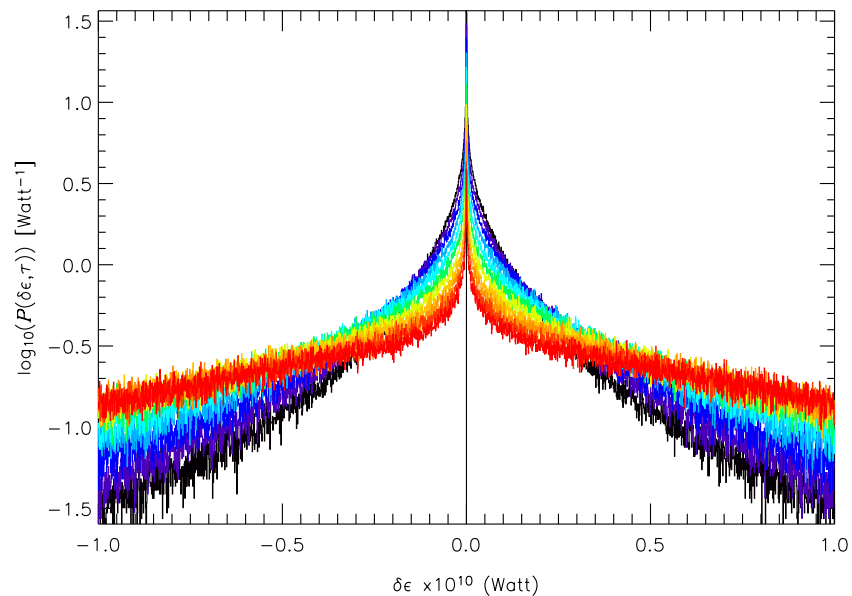

Fig. 1. Probability density functions (PDFs) of all the fluctuations in the solar wind $\varepsilon$ parameter for year 2000. The different curves correspond to time scales $\tau=64 \mathrm{~s}$ (black), $128 \mathrm{~s}, 256 \mathrm{~s}, \ldots$, $65536 \mathrm{~s}(18.2 \mathrm{~h})(\mathrm{red})$. The number of available $\delta \varepsilon$ values varied from 436303 for $\tau=1024 \mathrm{~s}$ to 457608 for $\tau=16536 \mathrm{~s}$. A bin size of $0.001^{10} \mathrm{~W}$ was used for $\delta \varepsilon$ in this plot.

in GSM co-ordinates at 16-s resolution. The IMF data were re-sampled at 64-s resolution, and then used to construct a single time series of the solar wind $\varepsilon$ parameter which provides a measure of the fraction of solar wind Poynting flux entering the magnetosphere via magnetic reconnection:

$\varepsilon=v_{S W} \frac{B^{2}}{\mu_{0}} l_{0}^{2} \sin ^{4}\left(\frac{\theta}{2}\right)$

where $\quad B=\left(B_{x}^{2}+B_{y}^{2}+B_{z}^{2}\right)^{1 / 2}, \quad \mu_{0}=4 \pi \times 10^{7} \mathrm{H} \mathrm{m}^{-1}$, $l_{0}=7 R_{E}$, and $\theta=\arctan \left(\frac{\left|B_{y}\right|}{B_{z}}\right)$.

This parameter was not evaluated using measurements made when ACE was within the magnetosphere.

The calculation of scaling parameters for $\varepsilon$ is known to be problematic (Hnat et al., 2002b). Equation (1) shows that $\varepsilon$ is a minimum (maximum) when the angle $\theta=0^{\circ}\left(180^{\circ}\right)$, corresponding to the IMF pointing directly northward (southward). The problem arises in the $\sin ^{4}(\theta / 2)$ term which ensures the distribution of $\varepsilon$ values is dominated by many small, effectively near zero values, and then it decays rapidly, converging asymptotically toward zero for $\varepsilon \sim 10^{10} \mathrm{~W}$ and greater. That is, weak solar wind coupling is prevalent, and intense coupling is rare.

\subsection{Scaling collapse of the fluctuation PDFs}

Fluctuations in $\varepsilon$ were analysed to elucidate the techniques we use to quantify the fractal qualities of the data. The results also provided context for our main analysis of $v_{\mathrm{LOS}}$ fluctuations which may be partly driven by the $\varepsilon$ fluctuations. The first technique aims to identify a single component, power law scaling regime of $\varepsilon$ fluctuations through an analysis of

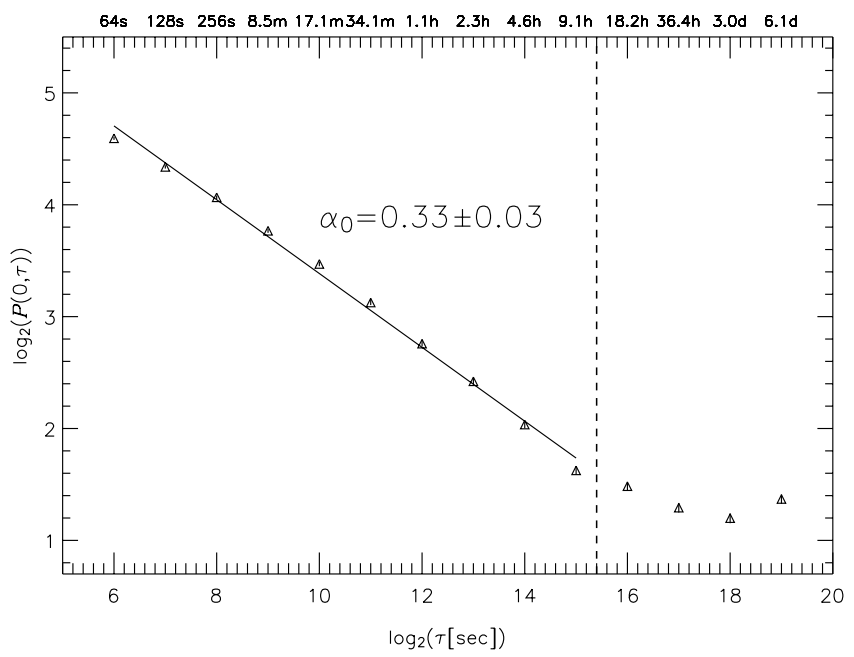

Fig. 2. Scaling of the PDF peaks, $\log _{2} P(0, \tau)$ versus $\log _{2} \tau$, for the $\delta \varepsilon$ curves shown in Fig. 2 . The linear best fit to the data yields a slope of $\alpha_{0}=0.33 \pm 0.03$ using a bin size of $\Delta \delta \varepsilon=0.001^{10} \mathrm{~W}$. The error bars assume shot noise $\propto \sqrt{n} / n$, and the vertical dashed line indicates the approximate outer time scale for the power law regime.

probability density functions (PDFs). We define a PDF element as $\delta n /(n \Delta x)$ where $n$ is the total number of samples, and $\delta n$ is the number of samples within bins of size $\Delta x$. The subsequent account is concise, following the detailed accounts given by Hnat et al. (2002a, b), and inspired by the earlier work of Mantegna and Stanley (1995).

A fluctuation in $\varepsilon$ was defined as $\delta \varepsilon(t, \tau)=\varepsilon(t)-\varepsilon(t-\tau)$ where $\tau$ is an adjustable time separation between samples. In practice, every $\varepsilon_{i}^{t h}$ sample was tested for the existence of an $\varepsilon$ fluctuation $\delta \varepsilon_{i}=\varepsilon_{i}-\varepsilon_{j}$ such that the actual time separation between samples, $\delta t$, equaled one of the native time series values $\tau=64 \mathrm{~s}, 128 \mathrm{~s}, 256 \mathrm{~s}, \ldots$, or $524288 \mathrm{~s}$ (roughly $1 \mathrm{~min}$ to 6.1 days). A $2 \%$ relative error in $|\delta t-\tau|$ was tolerated, but the sample corresponding to the minimum difference between $\delta t$ and $\tau$ was always identified. In practice, the average relative error in $|\delta t-\tau|$ was $\ll 1 \%$. Our search algorithm had the advantage of recovering many fluctuations at large $\tau$ from relatively small data sets, yet the disadvantage of introducing correlations between samples at the largest $\tau$. Even so, our calculations are in agreement with previous results.

Figure 1 shows the PDFs of $\delta \varepsilon$ on a logarithmic scale for various values of $\tau$ ranging from $64 \mathrm{~s}$ (black) to $18.2 \mathrm{~h}$ (red). That is, we plot $\log _{10} P(\delta \varepsilon, \tau)$ versus $\delta \varepsilon$, where $P(\delta \varepsilon, \tau)=\delta n /(n \Delta \delta \varepsilon)$ is the occurrence of $\delta \varepsilon$ in bins of size $\Delta \delta \varepsilon$. The PDFs of $\delta \varepsilon$ are symmetric, peaking at the origin, and then gradually decaying toward zero for large positive and negative values. However, there is a greater occurrence of small (large) $\delta \varepsilon$ values for small (large) values of $\tau$. The crossover points are located near $\delta \varepsilon \sim \pm 0.3^{10} \mathrm{~W}$. Clearly, these PDFs have the long tails characteristic of strongly leptokurtic, non-Gaussian functions. 


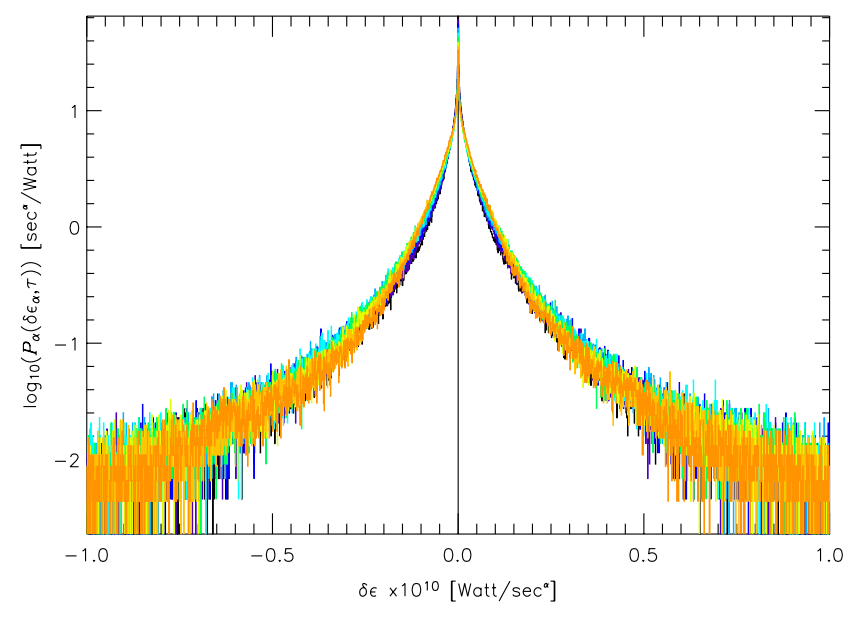

Fig. 3. Scaling collapse of the PDFs of $\delta \varepsilon$ for $\tau=64 \mathrm{~s}$ to $32768 \mathrm{~s}$ (9.1 h) shown in Fig. 1 onto a common curve (see text). A single scaling exponent $\alpha_{0}=0.33$ was used (Fig. 2), but the scaling collapse was also good for a range of values including $\alpha_{0}=0.43$.

Figure 2 plots the logarithm of the peak probability of each PDF versus the logarithm of $\tau$. That is, we plot estimates of $\log _{2} P(0, \tau)$ versus $\log _{2} \tau$. The $P(0, \tau)$ values were estimated by averaging the four values of $P(\delta \varepsilon, \tau)$ centred on $\delta \varepsilon=0$, and using bins of width $\Delta \delta \varepsilon=0.001^{10} \mathrm{~W}$. Within statistical error, the data points lie on a straight line with slope $\alpha_{0}=0.33 \pm 0.03$ over 9 octaves of $\tau$ ranging from $64 \mathrm{~s}$ to $\sim 9.1 \mathrm{~h}$. Clearly, the linearity of the data breaks down at $\tau \sim 18.2 \mathrm{~h}$, and definitely for $\tau \geq 36.4 \mathrm{~h}$. The vertical line in Fig. 2 represents the approximate limit of the turbulent regime sharing the same scaling (and non-linear dynamics). The single, power law scaling parameter $\alpha_{0}$ will be used to re-scale the PDFs shown in Fig. 1.

Figure 3 shows collapse of all the PDFs of $\delta \varepsilon_{s}$ from $\tau=64 \mathrm{~s}$ to $9.1 \mathrm{~h}$ (cf. Fig. 1) onto a single, common curve. Here, $\log _{10}\left(P_{s}\left(\delta \varepsilon_{s}, \tau\right)\right)$ versus $\delta \varepsilon_{s}$ where $P_{S}\left(\delta \varepsilon_{s}, \tau\right)=\tau^{\alpha_{0}} P(\delta \varepsilon$, $\tau)$ and $\delta \varepsilon_{s}=\delta \varepsilon \tau^{-\alpha_{0}}$ has been plotted. Most of the PDF for $\tau=18.2$ (not shown) also collapsed to the same curve, but systematic differences began to show, and the scaling break was indisputable by $\tau=36.4 \mathrm{~h}$. The choice of a small bin size, $\Delta \delta \varepsilon=0.001^{10} \mathrm{~W}$, helped to emphasise the scatter in our estimates of PDF elements. The scatter bars assuming shot noise within individual bins are only slightly larger than the scatter shown. Within statistical error, we conclude the results show single scaling exponent collapse of fluctuations in $\varepsilon$ for all $\tau$ from $64 \mathrm{~s}$ to $9.1 \mathrm{~h}$.

Scaling collapse of the solar wind $\varepsilon$ parameter has been shown previously (e.g., Hnat et al., 2002a, b). Using Wind spacecraft observations made during 1995 to 1998 , Hnat et al. (2002b) showed the peaks of the PDFs of $\delta \varepsilon$ were well described by a power law for $\tau$ up to $4.6 \mathrm{~h}$, and possibly even for $\tau=9.2 \mathrm{~h}$ and more (see their Fig. 2). Hnat et al. (2003b) also showed the solar wind parameter $v B^{2}$ scaled for $\tau$ up to $26 \mathrm{~h}$.

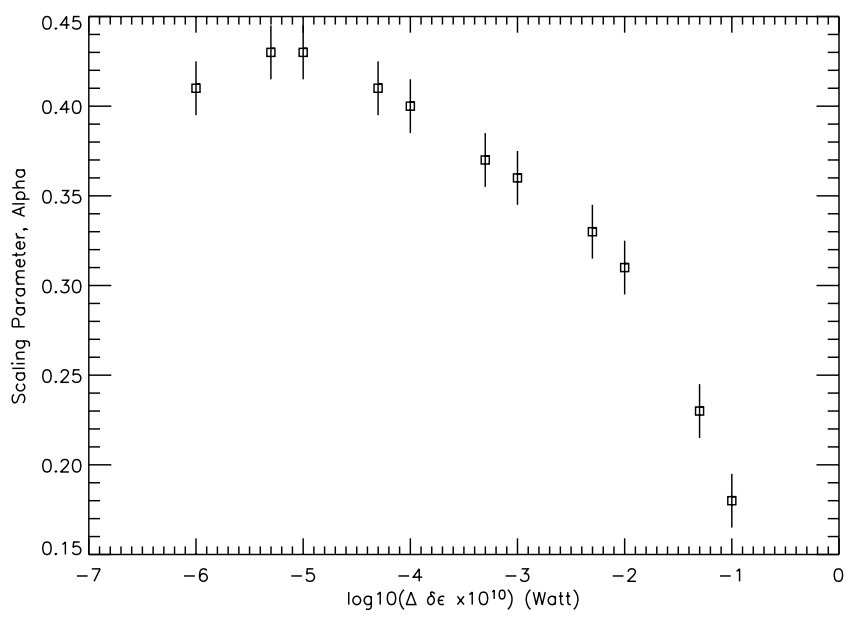

Fig. 4. The variation of the scaling parameter $\alpha_{0}$ estimated using the peaks of the PDFs plotted against the bin size used to resolve the amplitude and shape of the peaks. A local maximum of $\alpha_{0}=0.43 \pm 0.03$ was obtained for a bin size of about $\Delta \delta \varepsilon \sim 0.00001^{10} \mathrm{~W}$ (i.e. $\log _{10}=-5$ on the scale).

Given we expect solar cycle variability (Pagel and Balogh, 2002; Hnat et al., 2005) and a particular turbulent regime may not end suddenly, our outer temporal scale, $\tau \approx 9.1 \mathrm{~h}$, is in basic agreement. However, Hnat et al. (2002b) obtained $\alpha_{0}=0.42 \pm 0.03$ using bin sizes $\Delta \delta \varepsilon$ which decrease logarithmically with $\delta \varepsilon$, and this disagrees with our initial estimate of $\alpha_{0}=0.33 \pm 0.03$.

The amplitude and shape of the peaks is controlled by the choice of bin size for these strongly leptokurtic PDFs with $\delta$-function like peaks. Figure 4 illustrates how the scaling parameter $\alpha_{0}$ varied with the bin size $\Delta \delta \varepsilon$. It can be seen that $\alpha_{0}$ had a local maximum of $\alpha_{0}=0.43 \pm 0.03$ for $\Delta \delta \varepsilon \sim 0.00001^{10} \mathrm{~W}$, in agreement with earlier work. On the one hand, it makes sense to estimate the scaling parameter $\alpha_{0}$ using the peaks where there is the largest number of $\delta \varepsilon$ values and thus statistical fluctuations are least. On the other hand, errors in the measurements of $B_{x}, B_{y}, B_{z}$, and $v_{s w}$ combine in a complicated way to limit the accuracy of estimates of $\varepsilon$. In turn, these errors affect estimates of the smallest values of $\delta \varepsilon$ and also the shape of the peaks. The local maximum in Fig. 4 may correspond to the point where the effects of numerical and measurement errors begin to dominate for smaller bin sizes.

Hnat et al. (2002b) identified the similarity of $\alpha_{0}$ for PDFs of $\delta \varepsilon$ and fluctuations in the auroral electrojet indices, AL, $\mathrm{AU}$, and AE. This raised the possibility that small-scale fluctuations in the magnetospheric current system are directly driven by the solar wind. Figure 4 illustrates how difficult it is to scale the $\varepsilon$ parameter, and suggests caution in making direct comparisons between the absolute values of $\alpha_{0}$ obtained for completely different data sets. However, the presence of fluctuations common to the solar wind and electrojet indices 


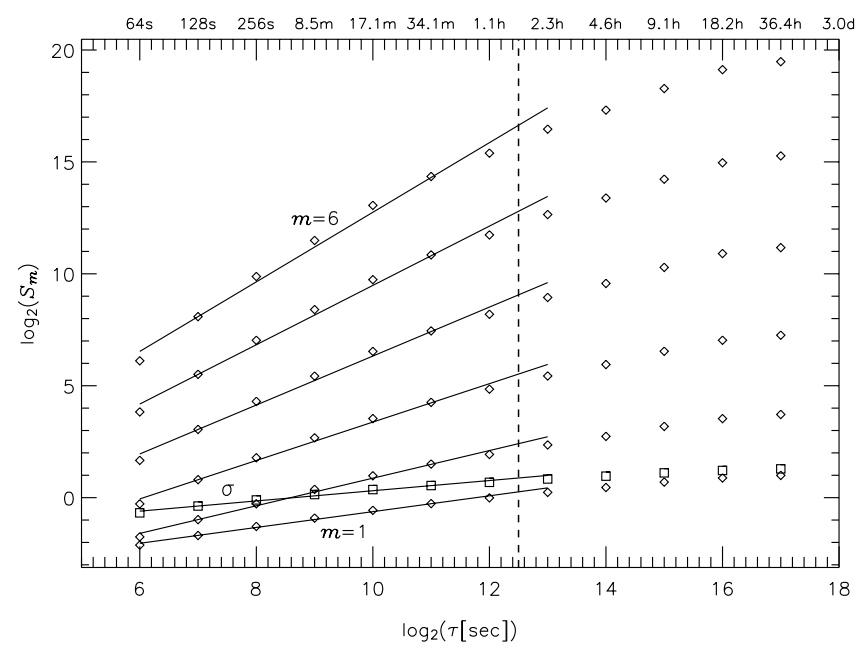

Fig. 5. A generalised structure function analysis (GSF) showing the variation of the moments $S_{m}(\tau)=\left\langle|\delta \varepsilon(t, \tau)|^{m}\right\rangle$ with time scale $\tau$ for orders $m=1$ to 6 . The results are linearised on a log-log scale over time scales from $64 \mathrm{~s}$ to $\sim 2 \mathrm{~h}$. We also show the variation of the standard deviation $\sigma(\tau)=\left[S_{2}(\tau)\right]^{1 / 2}$ with $\tau$ (squares). Least squares fits have been applied to all of the moments.

can be inferred from relative variations in the corresponding scaling parameters, such as those which depend upon solar phase (Hnat et al., 2005).

\subsection{Generalised Structure Function (GSF) analysis}

The previous analysis identified a small-scale solar wind regime where single exponent scaling exists. Although $P_{s}\left(\delta \varepsilon_{s}, \tau\right)$ collapse suggests self-affinity, there may be nonself-affine fluctuations which also collapse the PDFs. Ideally, the higher order moments, including the standard deviation, should be analysed to confirm self-affinity (Hnat et al., 2002b, 2003a). Here we use generalised structure functions (GSF), or "variograms," $S_{m}$, to better characterise the leptokurtic properties of the data. As explained by Hnat et al. (2003a, 2005), the variograms of the fluctuations $\delta \varepsilon(t, \tau)=\varepsilon(t)-\varepsilon(t-\tau)$ are defined as the time average of their moments, $S_{m}(\tau)=\left\langle|\delta \varepsilon(t, \tau)|^{m}\right\rangle$ where $m$ is any real number, not necessarily positive. For power law scaling of $\delta \varepsilon(t, \tau)$, $S_{m}(\tau) \propto \tau^{\zeta(m)}$, and a $\log -\log$ plot of $S_{m}$ versus $\tau$ will reveal a straight line for each $m$ with gradients $\zeta(m)$. If the time series of $\varepsilon$ is self-affine, then $\zeta(m)=\alpha_{\mathrm{GSF}} m$ with a single scaling exponent $\alpha_{\mathrm{GSF}}$. The parameter $\alpha_{\mathrm{GSF}}$ obtained in this way should also collapse the PDFs onto a common curve, as in Fig. 3.

Figure 5 shows the variogram of the moments $S_{m}(\tau)$ for $m=1$ to 6 (bottom to top). $S_{0}(\tau)=\left\langle|\delta \varepsilon|^{0}\right\rangle$ is trivial and not shown, $S_{1}(\tau)=\left\langle|\delta \varepsilon|^{1}\right\rangle$ is the mean, and $S_{2}(\tau)$ provides the standard deviation, $\sigma(\tau)=\left[S_{2}(\tau)\right]^{1 / 2}$, of the fluctuations. Within statistical error, the moments for $m=1$ to 6 are linear over 6 to 7 octaves of $\tau$ ranging from $64 \mathrm{~s}$ to $\sim 2 \mathrm{~h}$. This sug-

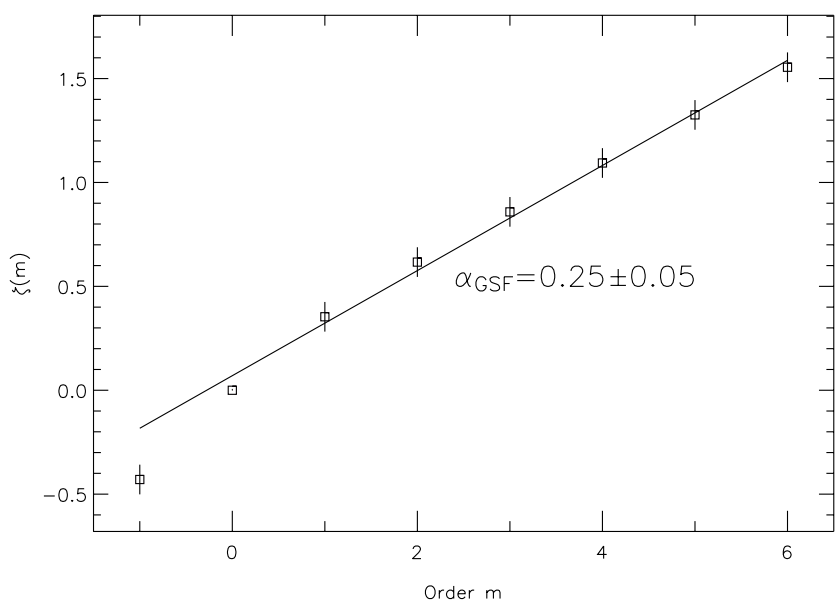

Fig. 6. This figure shows the gradients of the linear best fit curves, $\zeta(m)$, shown in Fig. 6, versus order $m$ over the range $m=-1$ to 7 . The gradient of the best fit curve to the present, combined results gives $\alpha_{\mathrm{GSF}}=0.25 \pm 0.05$.

gests the break in the scaling regime is somewhat less than the $\sim 9.1 \mathrm{~h}$ obtained using the preceding analysis. However, the estimates of the moments, especially for large absolute values of $m$, can be greatly perturbed by a few very large outliers. That is, the moments become very sensitive to statistical uncertainty created by the small number of very large fluctuations $\delta \varepsilon$ available at all values of $\tau$. This familiar problem was moderated by only calculating $S_{m}(\tau)$ using $\delta \varepsilon$ values less than $10 \sigma$, where $\sigma$ was initially estimated using all samples. This process is known as "conditioning." However, there is still a practical range of $m$ in the GSF analysis.

Figure 5 (squares) show the scaling of $\sigma(\tau) \propto \tau^{H}$ where $H$ is the Hurst exponent [ $\zeta(2) / 2]$. Like the results for the moment $S_{1}(\tau)$, within experimental uncertainty, the scaling of $\sigma(\tau)$ is linear out to at least $\tau=2.3 \mathrm{~h}$. We obtain $H=0.23 \pm 0.05$, but note that this parameter does not distinguish between Gaussian and non-Gaussian fluctuations (Mandelbrot, 2002).

In Fig. 6 we test whether $S_{m}(\tau) \propto \tau^{\zeta(m)}$, plotting $\zeta(m)$ versus order $m$ over the range $m=-1$ to 7 . By definition, $\zeta(0)=0$ with no error. Within experimental error, the gradients $\zeta(m)$ increase linearly with $m$, except for the case $m=-1$, the calculation of which is too sensitive to the problematic peaks of the PDFs. The gradient of the best fit curve gives $\alpha_{\mathrm{GSF}}=0.25 \pm 0.05$, and this value also collapsed the PDFs onto a single common curve (cf. Fig. 3). In fact, good scaling collapse was achieved over a fairly broad range of scaling parameters. Overall, the analysis of the higher order moments confirms the self-affinity of the $\varepsilon$ data over time scales of $64 \mathrm{~s}$ to $2 \mathrm{~h}$ or more.

Note that prior to the analysis of $\varepsilon$ fluctuations, the technique was exercised using a synthetic time series with well known properties. Using Eq. (2.3) of Janicki et al. (2005) an 


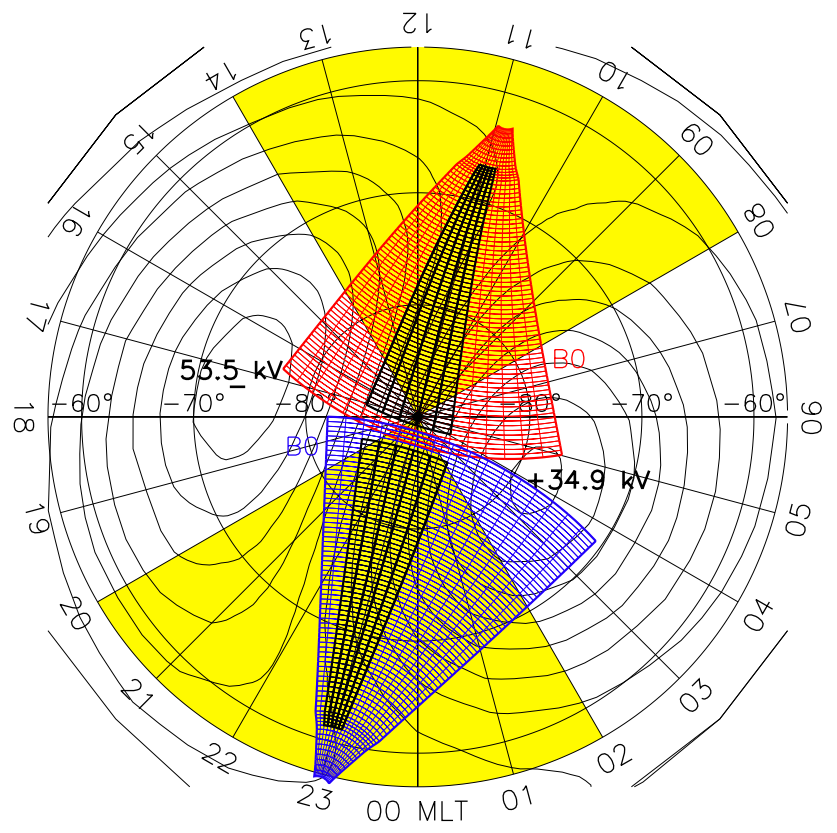

Fig. 7. The fields of view (FoV) of the Halley (top) and TIGER (bottom) SuperDARN radars are shown mapped to a clock dial plot of MLT and latitude. Beam 0 of each radar is the western most beam, and the observation cells used in this study are drawn in bold. Equipotentials given by the DMSP satellite-based Ionospheric Convection Model (DICM) (Papitashvili and Rich, 2002) for ( $B_{x}, B_{y}$, $\left.B_{z}\right)=(0,0,-5) \mathrm{nT}$ are also shown. The minimum electric potential in the dusk cell was $-53.3 \mathrm{kV}$, the maximum potential in the dawn cell was $34.9 \mathrm{kV}$, and contours are separated by $2.5 \mathrm{kV}$.

$\alpha$-stable Levy walk with $\alpha=1.5$ (not the same as $\alpha_{0}$ or $\alpha_{\mathrm{GSF}}$ ) was created. This time series exhibits behaviour reminiscent of economic index fluctuations (e.g. the S\&P 500, Mantegna and Stanley, 1995). Approximately one million samples were generated, and then analysed using peak scaling and GSF analysis. From peak scaling, $\alpha_{0}=0.63 \pm 0.02$ was obtained, and from GSF $H=0.50 \pm 0.02$ and $\alpha_{\mathrm{GSF}}=0.53 \pm 0.05$ were obtained. The equality $H=\alpha_{\mathrm{GSF}}$ is expected for these uncorrelated fluctuations. All the moments exhibited power law scaling over the full range of $\tau$, and scaling collapse of the PDFs was also achieved.

The preceding techniques can be used to quantify the characteristics of turbulent or SOC-like regimes, and will be applied to concurrent HF radar measurements in subsequent sections.

\section{Scaling of the SuperDARN $v_{\operatorname{LOS}}$ observations}

\subsection{Instruments and data set}

SuperDARN is a network of oblique sounding HF backscatter radars established to study convection in the high-latitude ionosphere (Greenwald et al., 1995). SuperDARN radars typically measure the line-of-sight Doppler velocity, $v_{\mathrm{LOS}}$, in up to 16 different beam directions separated by $3.24^{\circ}$ and at 75 range gates starting from $180 \mathrm{~km}$ and separated by $45 \mathrm{~km}$. Depending on group range, individual $v_{\mathrm{LOS}}$ measurements are averaged over an area of $\sim 100 \times 45 \mathrm{~km}$. Here we use observations made with the Halley SuperDARN radar $\left(75.5^{\circ} \mathrm{S}, 26.6^{\circ} \mathrm{W} ; 62^{\circ} \mathrm{CGM}\right)$, and the TIGER SuperDARN radar $\left(43.4^{\circ} \mathrm{S}, 147.2^{\circ} \mathrm{E}\right.$; $\left.55^{\circ} \mathrm{CGM}\right)$ (Dyson and Devlin, 2000; Dyson et al., 2003). Year 2000 was chosen for analysis, because this was the first full year of joint radar operations.

SuperDARN radars repeatedly transmit pulse sets to measure and average the autocorrelation function $(\mathrm{ACF})$ of returned signals, whilst minimising the effects of range aliasing out to great ranges $(\sim 3300 \mathrm{~km})$. The pulse set uses a fundamental lag length of $2400 \mu \mathrm{s}$, which corresponds to an effective Nyquist frequency of $\pm 208.3 \mathrm{~Hz}$. For a typical operating frequency of $12 \mathrm{MHz}$, this corresponds to a Nyquist velocity of $\pm 2604 \mathrm{~m} \mathrm{~s}^{-1}$. Hence, depending on the chosen pulse set and operating frequency, SuperDARN radars will alias large velocity transients of $2-3 \mathrm{~km} \mathrm{~s}^{-1}$ and more. If the ionospheric convection is fractal in space and time, the small-scale electric field structure will not be resolved.

An algorithm known as "FITACF" (Baker et al., 1995) uses the coherently averaged ACFs to estimate the backscatter power (signal-to-noise ratio) (dB), line-of-sight Doppler velocity $v_{\mathrm{LOS}}\left(\mathrm{m} \mathrm{s}^{-1}\right)$, and Doppler spectral width $\left(\mathrm{m} \mathrm{s}^{-1}\right)$. Only ionospheric scatter with FITACF power $>3 \mathrm{~dB}$ and $v_{\text {LOS }}$ error $<200 \mathrm{~m} \mathrm{~s}^{-1}$ were analysed here. The velocity errors generally increased with decreasing power and increasing spectral width. Thresholding the data with a large velocity error ensured a sufficient number of samples with large velocity. However, when the error threshold was reduced, our results were basically the same, except scatter in the PDFs increased, especially for the rare fluctuations of most interest. As will be explained, the FITACF velocity errors cannot explain the long tails of the PDFs of $\delta v_{\text {LOS }}$ to be shown.

Figure 7 shows the field of view (FoV) of the Halley radar (blue) and TIGER (red) radar mapped to a standard clock-dial plot consisting of MLT and magnetic latitude measured in altitude adjusted corrected geomagnetic (CGM) coordinates (Baker and Wing, 1989). The Halley radar is located $\sim 6^{\circ}$ closer to the geomagnetic pole than the TIGER radar. Hence the Halley radar is better suited for observing the greater cusp (as previously defined), whereas TIGER is better suited for observing the nightside auroral oval. However, both radars can observe scatter on open and closed field lines, day or night.

The daytime sector was defined as the six-hour interval 08:00 to 14:00 MLT for the Halley radar, and the nighttime sector as 20:00 to 02:00 for the TIGER radar (yellow shading). The daytime sector was biased toward pre-noon because this was better aligned with the observed distribution of echoes with large spectral width, and to preserve symmetry 
with the nighttime sector used for TIGER. The latter was biased toward pre-midnight where the spectral width boundary is a better proxy for the open-closed field line boundary (Parkinson et al., 2004; Chisham et al., 2005). Note that we also aim to separate the analysis according to open and closed flux.

Ideally, the radar FoVs should have been fixed in MLT for all UT to ensure all of the $v_{\text {LOS }}$ fluctuations were purely temporal. Although the observed $v_{\text {LOS }}$ fluctuations were mostly due to temporal variations in the electric field (Abel and Freeman, 2002), the radars rotated through their respective time sectors once per day. Hence the observed fluctuations were influenced by spatial variations in the underlying statistical convection pattern, especially at the largest time scale $\tau$. Knowledge of the unique convection pattern at every instant would be required to correct for the spatial variations in our analysis. Even so, we might expect complimentary fluctuations in space and time.

Most of the ionospheric scatter detected by SuperDARN radars at ranges greater than $600 \mathrm{~km}$ is thought to emanate from F-region decametre-scale irregularities which drift at the $\boldsymbol{E} \times \boldsymbol{B} / B^{2}$ velocity (Villain et al., 1985). Hence fluctuations in $v_{\mathrm{LOS}}$ are proportional to fluctuations in the corresponding orthogonal electric field in the ionosphere. A fluctuation of $v_{\mathrm{LOS}}=200 \mathrm{~m} \mathrm{~s}^{-1}$ corresponds to a fluctuation of $\boldsymbol{E} \approx 8.8 \mathrm{mV} \mathrm{m}^{-1}$ at representative group ranges within the Halley radar FoV. The magnetic field is stronger within the TIGER FoV, so the same $v_{\mathrm{LOS}}$ fluctuation corresponds to a fluctuation of $\boldsymbol{E} \approx 11.3 \mathrm{mV} \mathrm{m}^{-1}$. Of course, the full vector electric fields will be somewhat larger on average.

Ionospheric echoes with low spectral width $\leq 75 \mathrm{~m} \mathrm{~s}^{-1}$ are defined as "Population A", and those with large spectral width $\geq 150 \mathrm{~m} \mathrm{~s}^{-1}$ as "Population B" (Parkinson et al., 2003). Two widely separated thresholds were used to help resolve the two populations because of overlap between their corresponding PDFs. The two populations are often separated by a distinct boundary in latitude, which is also a reasonable proxy for the open-closed magnetic field line boundary in proximity to the dayside cusp (Baker et al., 1995) and in the pre-midnight sector (Lester et al., 2001; Parkinson et al., 2002; Chisham et al., 2004). Although high spectral widths are concentrated on open field lines, they can also occur on closed field lines (Dudeney et al., 1998; Woodfield et al., 2002; Wild et al., 2004), especially just equatorward of the open-closed boundary. Similarly, although low spectral widths are concentrated on closed field lines deeper in the magnetosphere, they can also occur on open field lines.

It is possible to construct up to 16 beams $\times 75$ ranges $=$ 1200 time series of $v_{\text {LOS }}$ for each radar. However, here we only consider the measurements made on the "meridional" beams of Halley $(6,7,8,9$, and 10) and TIGER (2, 3, 4, 5, and 6) (bold in Fig. 1). This ensured our statistics were dominated by fluctuations in the zonal electric field, which tend to be the strongest component in the polar cap. Data taken on the two beams adjacent to the actual meridional beams $(8$ and 4) were used to increase the statistical significance of our calculations. The scatter was also restricted to range gates between 630 and $3330 \mathrm{~km}$ (bold) to eliminate most of the Eregion scatter which occurs at closer ranges, and the known bad ranges which occur at the furthest ranges, a limitation of the pulse set design. Thus we formed up to $5 \times 61=305$ time series of $v_{\text {LOS }}$ for each radar.

For each radar time series, every $i$ th velocity sample, $v_{\mathrm{LOS}, i}$, was tested for the existence of a velocity fluctuation $\delta v_{\mathrm{LOS}, i}=v_{\mathrm{LOS}, i}-v_{\mathrm{LOS}, j}$ such that the actual time separation between samples, $\delta t$, equaled one of $\tau=1,2,4, \ldots$, or $256 \mathrm{~min}$. As with the $\delta \varepsilon$ analysis, a $2 \%$ relative error in $|\delta t-\tau|$ was tolerated, but the relative errors were mostly $\ll 1 \%$. The time resolution of the radar measurements was typically 1 or $2 \mathrm{~min}$, though sometimes as good as $6 \mathrm{~s}$ during special campaigns of limited duration. Hence our choice of a minimum $\tau=1 \mathrm{~min}$. The outer time scale of $256 \mathrm{~min}$ was limited by the 6-h windows of MLT (Fig. 7) and the Earth's rotation.

Each radar observation was tagged with an IMF vector determined by advecting the IMF measured at the spacecraft to the sub-solar magnetopause ( $\sim 10 \mathrm{Re}$ ) using hourly average solar wind speeds and the GSM $x$ distance. This simple method was adequate for a statistical analysis of numerous observations made over an entire year. Finally, the results for all chosen beams and ranges were compiled into single files, though separately for each radar and value of $\tau$. The results were further sorted according to the orientation of the IMF, MLT, and the Doppler spectral width.

\subsection{Results for Halley radar $v_{\mathrm{LOS}}$ (electric field)}

Figure 4 suggested caution in making a direct comparison between the absolute values of scaling parameters obtained for completely different data sets. However, direct comparison of scaling parameters should be possible when analysing subsets of the same measurements in a consistent way, including the choice of bin size, as will be done in the remainder of this paper. Nevertheless the results for the radar measurements need to be considered in the context of the results for $\varepsilon$ fluctuations, a possible driver for the $v_{\operatorname{LOS}}$ fluctuations.

Figure 8 shows PDFs of $v_{\mathrm{LOS}}$ for $\tau=1,2,4, \ldots, 256 \mathrm{~min}$ observed using the Halley radar in the noon-sector ionosphere. Part (a) shows the results for Population B (spectral width $\geq 150 \mathrm{~m} \mathrm{~s}^{-1}$ ), and part (b) shows the results for Population A (spectral width $\leq 75 \mathrm{~m} \mathrm{~s}^{-1}$ ). Population B echoes are more likely to be found at a higher latitude, on open field lines in the polar cap ionosphere. This was confirmed by an analysis of the PDFs of group range for these echoes. Doppler spectral width tends to increase with the amplitude of velocity fluctuations which are expected to increase in proportion to the mean flow speed for turbulence, so it is not surprising that larger $v_{\mathrm{LOS}}$ tended to be observed for Population B. Approximately $23 \%$ of $\left|v_{\mathrm{LOS}}\right|$ values were greater than $500 \mathrm{~m} \mathrm{~s}^{-1}$. 


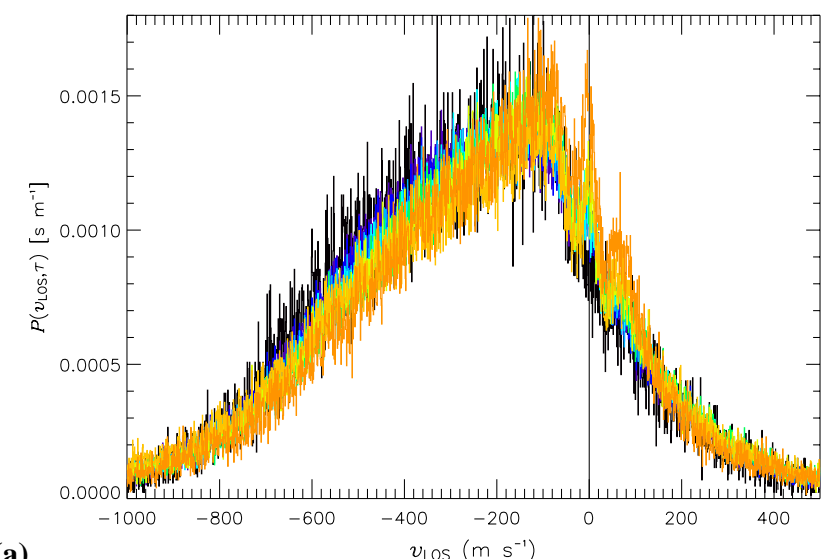

(a)

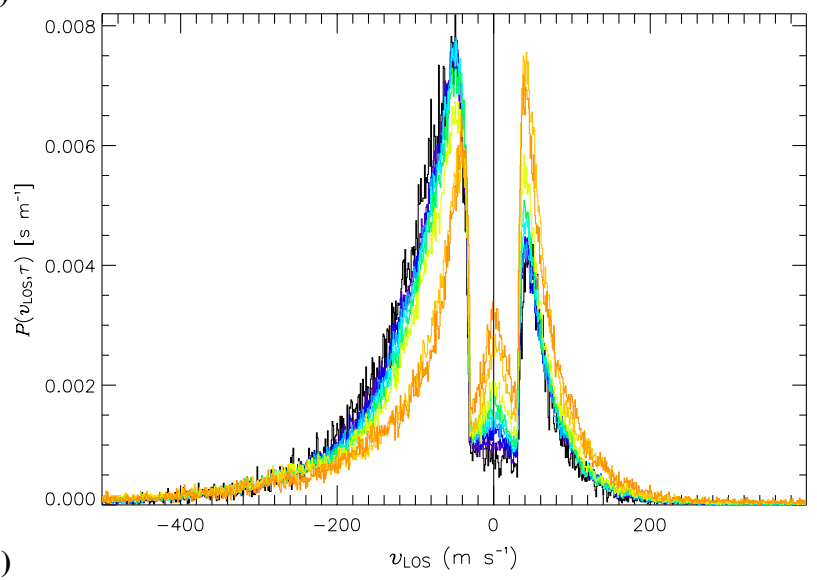

Fig. 8. PDFs of $v_{\mathrm{LOS}}$ for $\tau=1 \mathrm{~min}$ (black), $2 \mathrm{~min}, \ldots, 256 \mathrm{~min}$ (orange) observed using the Halley radar during 08:00 to 14:00 MLT throughout year 2000. The results were sorted according to (a) spectral width $\geq 150 \mathrm{~m} \mathrm{~s}^{-1}$ (Population B), and (b) spectral width $\leq 75 \mathrm{~m} \mathrm{~s}^{-1}$ (Population A). The number of samples varied from 86801 for $\tau=1 \mathrm{~min}$ to 562542 for $\tau=8 \mathrm{~min}$ for Population B, and from 58276 for $\tau=1 \mathrm{~min}$ to 310551 for $\tau=8 \mathrm{~min}$ for Population A. A bin size of $1 \mathrm{~m} \mathrm{~s}^{-1}$ was used for $v_{\mathrm{LOS}}$. The scatter obtained for this bin size is an approximate indication of shot noise.

The PDFs of $v_{\text {LOS }}$ are consistent with the expected behaviour of statistical convection models (cf. Fig. 7). In both cases, they consist of three overlapping distributions. The dominant peaks were associated with flows away from the radar, or anti-sunward into the polar cap, whereas the secondary peaks were associated with flows toward the radar, or in the sunward direction. In Fig. 8b, the PDFs shifted toward negative values (anti-sunward flow) for $B_{z}$ negative, and toward positive values (sunward flow) for $B_{z}$ positive (not shown). There was less of a shift in velocity with $B_{z}$ for Fig. 8a because Population B echoes were concentrated in the polar cap, a region less affected by expansion and contraction of the convection pattern. However, the ratio of the amplitude of negative to positive $v_{\text {LOS }}$ peaks increased (decreased) for $B_{z}$ southward (northward) subsets.

Notice that the PDFs for $\tau=256 \mathrm{~min}$ (orange) are shifted further towards positive $v_{\mathrm{LOS}}$, more than is the case for

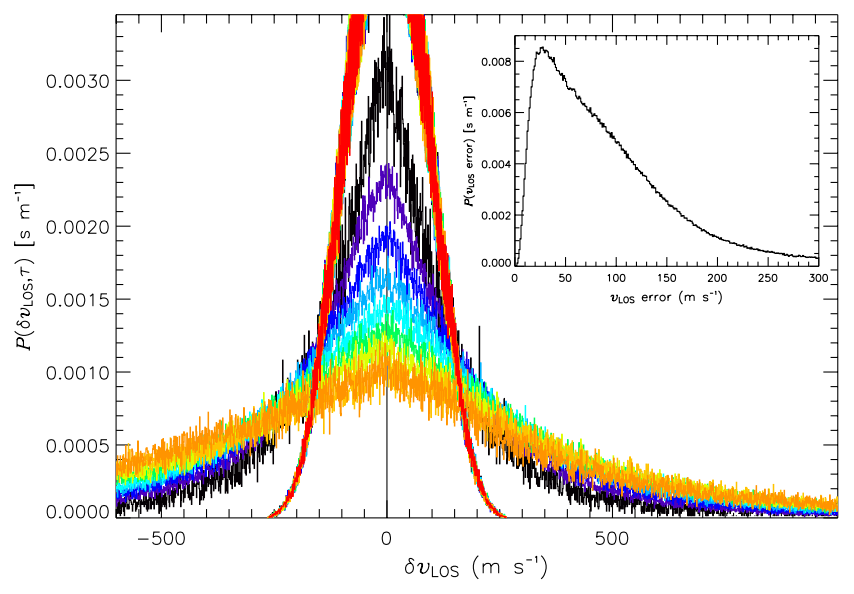

Fig. 9. PDFs of $\delta v_{\mathrm{LOS}}$ for $\tau=1 \mathrm{~min}$ (black), $2 \mathrm{~min}, \ldots, 256 \mathrm{~min}$ (orange) for Halley Population B echoes (main panel). The number of available samples varied from 86801 for $\tau=1 \mathrm{~min}$ to 562542 for $\tau=8 \mathrm{~min}$. The inset shows the PDF of the corresponding velocity errors. Bin sizes of $1 \mathrm{~m} \mathrm{~s}^{-1}$ were used for both PDFs.

shorter $\tau$. The PDFs were generated using $v_{\text {LOS }}$ values for which $\delta v_{\text {LOS }}$ values were identified. Hence the first possible value of $v_{\text {LOS }}$ occurred near 08:01 MLT for $\tau=1 \mathrm{~min}$, but not until 12:16 MLT for $\tau=256 \mathrm{~min}$. This ensured the time intervals used to calculate $\delta v_{\text {LOS }}$ were confined between 08:00 and 14:00 MLT. However, the $v_{\text {LOS }}$ samples for $\tau=256 \mathrm{~min}$ were biased toward the afternoon sector, 12:16 to 14:00 MLT, where slightly more flows with a sunward component were observed. Indeed, the PDFs for all time scales became more (less) alike for $B_{z}$ southward (northward).

Tertiary peaks were centred very close to $v_{\mathrm{LOS}}=0 \mathrm{~m} \mathrm{~s}^{-1}$ and correspond to echoes from zonal flows, especially at nearer ranges. The artificial-looking bite outs near $\pm 35 \mathrm{~m}$ $\mathrm{s}^{-1}$ are related to the behaviour of the algorithm which flags echoes as "ionospheric" or "ground" scatter. Because of TIGER's lower latitude, ionospheric echoes are more likely to be misidentified as ground scatter than vice versa. However, some of the small $v_{\text {LOS }}$ samples may have been caused by ground scatter misidentified as ionospheric scatter. Regardless, exclusion of these low Doppler echoes did not change the conclusions of this study, and improved methods for resolving the two kinds of scatter are under development.

Figure 9 shows the PDFs of $\delta v_{\mathrm{LOS}}(t, \tau)=v_{\mathrm{LOS}}(t)-$ $v_{\mathrm{LOS}}(t-\tau)$ for $\tau=1$ to $256 \mathrm{~min}$ for Halley Population B echoes. The PDFs reveal the extent to which large fluctuations in $v_{\text {LOS }}$ are likely to occur on longer time scales. Many more small fluctuations in $v_{\mathrm{LOS}}$ occur for $\tau=1 \mathrm{~min}$, whereas many more large fluctuations in $v_{\mathrm{LOS}}$ occur for $\tau=256 \mathrm{~min}$. The cross over point is near $\sim 200 \mathrm{~m} \mathrm{~s}^{-1}$. Although the radars do not measure $v_{\mathrm{LOS}}>2-3 \mathrm{~km} \mathrm{~s}^{-1}$, the available results suggest that at least $0.07 \%$ (34\%) of $\delta v_{\text {LOS }}$ values exceeded $500 \mathrm{~m} \mathrm{~s}^{-1}$ for $\tau=1 \mathrm{~min}(256 \mathrm{~min})$. Note that these fractions might increase if smaller spatial regions could be 


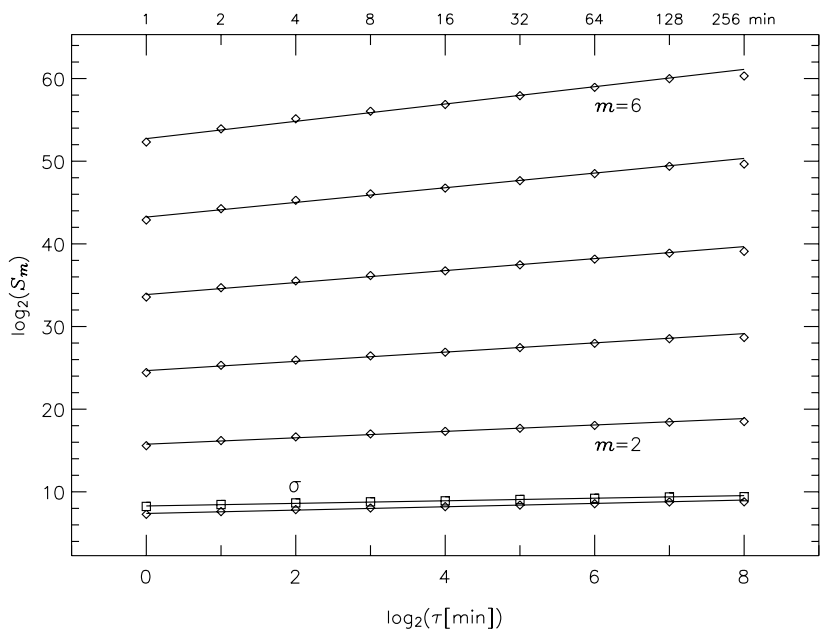

Fig. 10. A GSF analysis showing the variation of the moments $S_{m}(\tau)=\left\langle\left|\delta v_{\mathrm{LOS}}(t, \tau)\right|^{m}\right\rangle$ with time scale $\tau$ for orders $m=1$ to 6 . The results are linearised on a log-log scale over time scales from $1 \mathrm{~min}$ to $256 \mathrm{~min}$. Least squares fits have been applied and the error bars (barely visible) are standard deviations of the moments. The variation of the standard deviation $\sigma(\tau)=\left[S_{2}(\tau)\right]^{1 / 2}$ with $\tau$ is also shown (squares).

probed. Clearly, these PDFs have the long tails characteristic of leptokurtic functions.

A large velocity error threshold was used for data selection, even though large measurement errors are known to produce spurious "outliers." It might be argued that the leptokurtic PDFs of $\delta v_{\mathrm{LOS}}$ arose from measurement errors, but this is unlikely for the following reasons. In the first instance, the velocity errors are only weakly correlated with the velocities, so the measurement errors should be statistically independent of the time scale $\tau$. Hence if measurement errors completely dominated the tails, there would be no separation of the results according to $\tau$. Even so, the velocity errors might still exert a significant influence on the results, changing the shape of the PDFs in some way.

The insert of Fig. 9 shows the PDF of the velocity errors returned by FITACF for Halley Population B echoes. The most probable velocity error was $\sim 27 \mathrm{~m} \mathrm{~s}^{-1}$, but only $14 \%$ of the velocity errors were less than this, and there were many velocity errors greater than the most probable value; $38 \%(11 \%)$ of the velocity errors were greater than $100 \mathrm{~m}$ $\mathrm{s}^{-1}\left(200 \mathrm{~m} \mathrm{~s}^{-1}\right)$, respectively. This extreme distribution was used to create a discrete, synthetic time series with time resolution $1 \mathrm{~min}$ and duration 1 year. Variations in the time series had an occurrence frequency weighted by the corresponding probability density of errors out to the chosen threshold of $200 \mathrm{~m} \mathrm{~s}^{-1}$. Individual variations were given an amplitude of $2 / 3$ the velocity error, since the true error is always less than the maximum error. A random number generator was used to determine whether the individual variations were positive or negative, and to shuffle the final set of elements in time, but

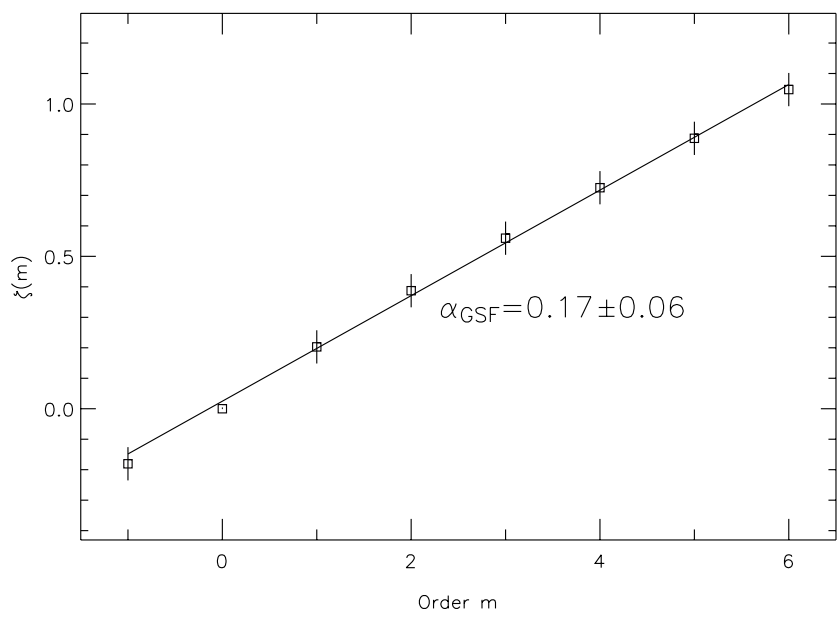

Fig. 11. This figure shows the gradients of the linear best fit curves, $\zeta(m)$ shown in Fig. 10, versus order $m$ over the range $m=-1$ to 6 . The gradient of the best fit curve to these gradients gives $\alpha_{\mathrm{GSF}}=0.17 \pm 0.06$.

the PDF of the variations was the same as in the inset (i.e. not Gaussian).

Next the synthetic time series was analysed in the same way as the actual measurements of $v_{\text {LOS }}$. The steep, bold red curve in the main panel of Fig. 9 shows the PDF of the synthetic time series for $\tau=256 \mathrm{~min}$, superimposed upon the synthetic results for all other $\tau$. As expected, the PDFs of the velocity errors are independent of $\tau$. Note that all the peaks are off scale. For $\delta v_{\text {LOS }}$ greater than $\sim 200 \mathrm{~m} \mathrm{~s}^{-1}$, it can be seen that the probability density for the synthetic data is very small compared to the actual radar measurements. The velocity errors cannot reproduce the long tails distinguishing the leptokurtic functions. In fact, the model results suggest the velocity errors had their greatest impact on the peaks, obscuring their $\delta$-function like shape.

Finally, note that the same leptokurtic tails were obtained when using velocity errors less than $200 \mathrm{~m} \mathrm{~s}^{-1}$ to threshold the data. Moreover, the long tails could not be reproduced by simply including velocities corresponding to errors $>200 \mathrm{~m}$ $\mathrm{s}^{-1}$ in the synthetic data. Also, the PDF of velocity errors had the largest number of large values for Halley Population B, so the most extreme case has been considered. However, there is an unlikely caveat to this argument: the FITACF velocity errors themselves may have been grossly under-estimated and poorly characterised.

Having shown the PDFs of $\delta v_{\text {LOS }}$ are indicative of geophysical behaviour, the extent to which the PDFs of $\delta v_{\mathrm{LOS}}$ collapse on to a common curve was investigated. The same methods used to achieve scaling collapse of $\delta \varepsilon$ explained in Sect. 2 were used. First, the scaling parameters $\alpha_{0}$ were estimated from the gradient of a log-log plot of the peak probability density versus time scale $\tau$. Next, the scaling parameter $\alpha_{\mathrm{GSF}}$ was identified using GSF analysis, noting the outer 


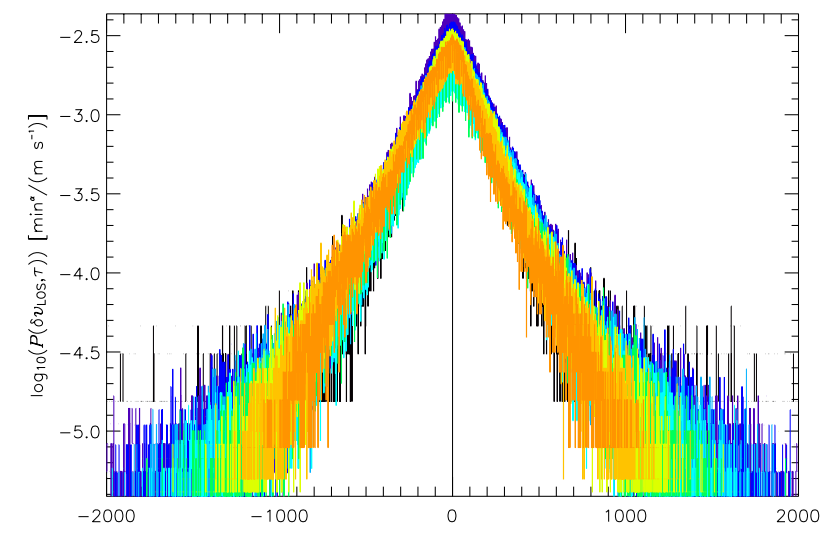

(a)

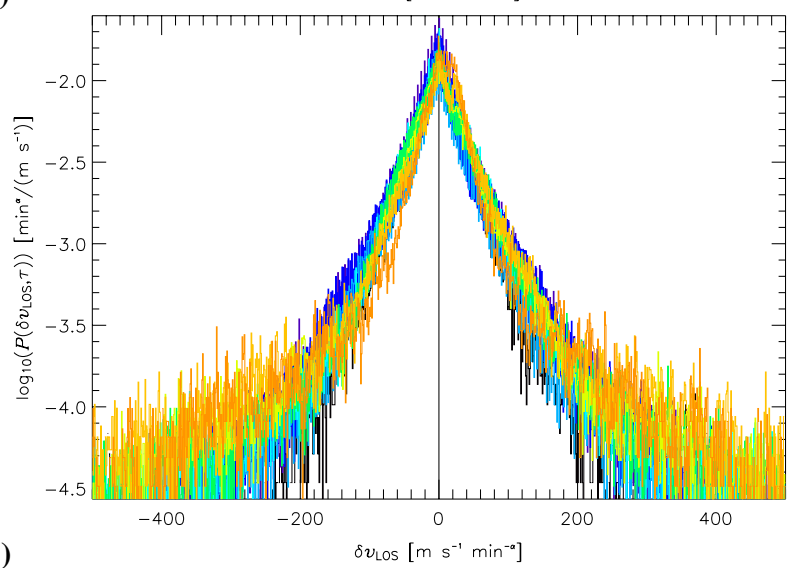

(b)

Fig. 12. The extent to which the PDFs of $\delta v_{\text {LOS }}$ collapse to a common curve for (a) Halley Population B, and (b) Halley Population A. The same method used to collapse the PDFs of $\delta \varepsilon$ was used. Common bin sizes of $1 \mathrm{~m} \mathrm{~s}^{-1}$ were used for $\delta v_{\text {LOS }}$ prior to scaling.

time scales of the self-similar regimes. For example, Fig. 10 shows the variogram of moments $S_{m}(\tau)=\left\langle\left|\delta v_{\operatorname{LOS}}(t, \tau)\right|^{m}\right\rangle$ for $m=1$ to 6 (bottom to top), as well as for the standard deviations, $\sigma(\tau)$ (squares). Figure 11 tests whether $S_{m}(\tau) \propto \tau^{\zeta(m)}$, plotting $\zeta(m)$ versus order $m$ over the range $m=-1$ to 6 (cf. Fig. 6). Within experimental error, the gradients $\zeta(m)$ increase linearly with $m$, and the gradient of the best fit curve gives $\alpha_{\mathrm{GSF}}=0.17 \pm 0.06$.

The GSF results were surprisingly linear, even for the higher order moments at the longest $\tau$. In part, this may be caused by our strongly conditioned data. The radars cannot measure $v_{\mathrm{LOS}}$ values out to $10 \sigma$. For example, for Halley Population B at $\tau=256 \mathrm{~min}, 10 \sigma$ corresponds to $6.8 \mathrm{~km} \mathrm{~s}^{-1}$, compared to the Nyquist limit of $\sim 2.6 \mathrm{~km} \mathrm{~s}^{-1}$. Hence $\delta v_{\text {LOS }}$ values less than $3 \sigma$ were used, but this only rejected a small fraction of the data because $3 \sigma$ is near to the Nyquist limit. Despite this difficulty, our analysis discerned consistent, relative variations in the scaling parameters amongst the different populations. Table 1 lists the values of $\alpha_{0}, \alpha_{\mathrm{GSF}}, H$, and their errors obtained for the different data sets. Although the absolute values of the different parameters differ, the relative
Table 1. Year 2000 scaling parameters obtained from PDF peaks and GSF analysis.

\begin{tabular}{lccc}
\hline Data set & $\alpha_{0}$ & $\alpha_{\mathrm{GSF}}$ & $H$ \\
\hline $\begin{array}{l}\text { ACE solar wind } \delta \varepsilon \\
\text { Halley dayside Population B, } \\
\text { spectral width }>150 \mathrm{~m} \mathrm{~s}^{-1}\end{array}$ & $0.43 \pm 0.03$ & $0.25 \pm 0.05$ & $0.23 \pm 0.05$ \\
$\begin{array}{l}\text { Halley dayside Population A, } \\
\text { spectral width }<75 \mathrm{~m} \mathrm{~s}^{-1}\end{array}$ & $0.23 \pm 0.04$ & $0.17 \pm 0.06$ & $0.16 \pm 0.05$ \\
$\begin{array}{l}\text { TIGER nightside Population B, } \\
\text { spectral width }>150 \mathrm{~m} \mathrm{~s}^{-1}\end{array}$ & $0.18 \pm 0.04$ & $0.12 \pm 0.06$ & $0.25 \pm 0.04$ \\
$\begin{array}{l}\text { TIGER nightside Population A, } \\
\text { spectral width }<75 \mathrm{~m} \mathrm{~s}^{-1}\end{array}$ & $0.35 \pm 0.04$ & $0.21 \pm 0.06$ & $0.17 \pm 0.05$ \\
\hline
\end{tabular}

variations are in good agreement. In all cases, the individual data points were reasonably close to the best fit straight lines.

Next we show the extent to which the PDFs for different $\tau$ collapse to a single common curve using the scaling parameters $\alpha_{0}$ given in Table 1 . Figure 12a plots $\log _{10}\left(P_{S}\left(\delta v_{\mathrm{LOS}}\right.\right.$, $\tau)$ ) versus $\delta v_{\mathrm{LOS} s}$ where $P_{s}\left(\delta v_{\mathrm{LOS}}, \tau\right)=\tau^{-\alpha_{0}} P\left(\delta v_{\mathrm{LOS}}, \tau\right)$, and $\delta v_{\mathrm{LOS} s}=\delta v_{\mathrm{LOS}} \tau^{-\alpha_{0}}$ for Halley Population B. The figure shows the collapse of the PDFs to the same curve is very good out to at least $\delta v_{\mathrm{LOS}}= \pm 800 \mathrm{~m} \mathrm{~s}^{-1}$ for all $\tau$. However, beyond this speed there is a slight "role-off" of the wings, especially for $\tau=256 \mathrm{~min}$. The mapping $\delta v_{\mathrm{LOS} s}=\delta v_{\mathrm{LOS}} \tau^{-\alpha_{0}}$ implies that the role-off corresponds to measured velocities of $\sim 2.5 \mathrm{~km} \mathrm{~s}^{-1}$, the approximate Nyquist limit of the radars. Hence the role-off is an artifact associated with under-sampling. Otherwise the scaling collapse is good, confirming the self-affinity of the $\delta v_{\text {LOS }}$ data over time scales of $60 \mathrm{~s}$ to $\sim 4 \mathrm{~h}$. In fact, as will be seen, this was the best scaling collapse achieved for any of the radar data sets.

Figure $12 \mathrm{~b}$ shows the scaling collapse for Halley Population A was good out to $\delta v_{\mathrm{LOS}}= \pm 250 \mathrm{~m} \mathrm{~s}^{-1}$ for $\tau=1$ to $128 \mathrm{~min}$, but started to fail for $\tau=256 \mathrm{~min}$ (orange). There was a slight bias for $v_{\text {LOS }}$ to increase (i.e. positive $\delta v_{\text {LOS }}$ ) at the longest time scale, $\tau=256 \mathrm{~min}$. The results are very noisy beyond $\delta v_{\mathrm{LOS}}= \pm 250 \mathrm{~m} \mathrm{~s}^{-1}$ since the electric fields tend to be much weaker deeper inside the magnetosphere. In Fig. 12a, there was also a slight bias for $v_{\mathrm{LOS}}$ to decrease at the longest time scale, $\tau=256 \mathrm{~min}$. When the measurements for Populations A and B were combined, the biases towards positive $\delta v_{\text {LOS }}$ for small $\left|\delta v_{\text {LOS }}\right|$, and towards negative $\delta v_{\text {LOS }}$ for large $\left|\delta v_{\text {LOS }}\right|$, remained. These biases are statistically significant because there were over 400000 samples at $\tau=256 \mathrm{~min}$.

Measurements of $\delta v_{\mathrm{LOS}}$ at the longest time scales, $\tau \geq 256 \mathrm{~min}$, represent a mixture of spatial and temporal variations. The $\delta v_{\text {LOS }}$ values represent differences between velocities measured in the morning and afternoon convection cells. The bias for larger $v_{\mathrm{LOS}}$ to decrease for Population $\mathrm{B}$ means the receding velocities were stronger (i.e. more negative) in the afternoon side of the convection throat at 
higher latitudes, whereas the bias for smaller $v_{\mathrm{LOS}}$ to increase for Population A means the receding velocities were weaker (i.e. less negative) in the afternoon side of the convection throat at lower latitudes. The biases which developed at longer $\tau$ were caused by spatial variations in the underling statistical convection pattern.

In the common modes of operation, SuperDARN radars measure $v_{\text {LOS }}$ on all 16 beams every $1 \mathrm{~min}(2 \mathrm{~min})$ using 3-s (6-s) coherently averaged ACFs (i.e. integrations). The $\delta v_{\text {LOS }}$ samples calculated at $\tau=1$ min mostly used measurements of $v_{\text {LOS }}$ made with 3-s integrations, whereas the $\delta v_{\text {LOS }}$ samples for $\tau \geq 2$ min used measurements made with a mixture of 3 - and 6 -s integrations. This is relevant because when the ionospheric irregularities and $v_{\text {LOS }}$ change on time scales comparable to $1-10 \mathrm{~s}$, slightly different $v_{\text {LOS }}$ values will be measured using different integration times. The results obtained for $\tau=1 \mathrm{~min}$ differ slightly because of this reason.

When the PDFs of Halley data shown in Fig. 12 were further sorted according to IMF $B_{z}$, the same scaling collapse occurred, except the results had more scatter because of the fewer number of samples. However, the PDFs of Population A had noticeably sharper peaks for $B_{z}$ southward, and broader peaks for $B_{z}$ northward. That is, there was a greater probability for large $\delta v_{\mathrm{LOS}}$ at all $\tau$ when $B_{z}$ was northward for Population A found at lower latitudes. Very minor changes were found when sorting the PDFs according to $B_{y}$. Table 2 shows the scaling parameter $\alpha_{0}$ versus the orientation of $B_{z}$.

Table 2 also lists the kurtosis of the unscaled PDFs averaged over $\tau=1$ to $256 \mathrm{~min},<\kappa>$. The kurtosis is given by:

$\kappa=\frac{1}{N} \sum_{j=0}^{N-1}\left[\frac{\left(\delta v_{\mathrm{LOS} j}-\overline{\delta v_{\mathrm{LOS}}}\right)}{\text { variance }^{1 / 2}}\right]^{4}-3$

(Spiegel and Boxer, 1972). When the kurtosis is negative, zero, and positive, the distributions are platykurtic, mesokurtic (Gaussian), and leptokurtic, respectively. Here all the kurtosis values were large and positive, and they decreased as $\tau$ increased (cf. Fig. 9). This confirms the PDFs were leptokurtic, having sharp peaks and long tails. The kurtosis was strongest for Population A, especially for $B_{z}$ southward. Moreover, the average kurtosis values were even larger when the PDFs for $\tau=64$ to $256 \mathrm{~min}$ were not included, thereby eliminating biases due to spatial variations in the large-scale convection.

Lastly, Table 2 also lists root-mean-square (RMS) values of the velocity fluctuations averaged over $\tau=1$ to $256 \mathrm{~min}$, $<\delta v_{\mathrm{RMS}}>$. These RMS values facilitate inter-comparison between the magnitude of the velocity fluctuations observed by the two radars. Consistent with Fig. 9, $\delta v_{\mathrm{RMS}}$ increased with $\tau$ for every way the results were sorted. Also, $<\delta v_{\mathrm{RMS}}>$ was larger for Population $\mathrm{B}$ which had larger $v_{\mathrm{LOS}}$. It is important to note that $<\delta v_{\mathrm{RMS}}>$ was substantially smaller (greater) for $B_{z}$ southward (northward) for Population A, but not for Population B.
Table 2. Scaling parameters $\alpha_{0}$ for $v_{\text {LOS }}$ observed by the Halley radar in the dayside ionosphere.

\begin{tabular}{llcrc}
\hline $\begin{array}{l}\text { Population } \\
\text { (spectral width) }\end{array}$ & $B_{z}$ & $\alpha_{0}$ & $<\kappa>$ & $<\delta v_{\mathrm{RMS}}>$ \\
\hline $\mathrm{B}\left(>150 \mathrm{~m} \mathrm{~s}^{-1}\right)$ & All & $0.19 \pm 0.04$ & 6.7 & $497.3 \mathrm{~m} \mathrm{~s}^{-1}$ \\
$\mathrm{~B}\left(>150 \mathrm{~m} \mathrm{~s}^{-1}\right)$ & South & $0.18 \pm 0.04$ & 6.5 & $498.8 \mathrm{~m} \mathrm{~s}^{-1}$ \\
$\mathrm{~B}\left(>150 \mathrm{~m} \mathrm{~s}^{-1}\right)$ & North & $0.19 \pm 0.04$ & 6.9 & $482.6 \mathrm{~m} \mathrm{~s}^{-1}$ \\
$\mathrm{~A}\left(<75 \mathrm{~m} \mathrm{~s}^{-1}\right)$ & All & $0.23 \pm 0.04$ & 58.2 & $248.9 \mathrm{~m} \mathrm{~s}^{-1}$ \\
$\mathrm{~A}\left(<75 \mathrm{~m} \mathrm{~s}^{-1}\right)$ & South & $0.23 \pm 0.04$ & 76.2 & $203.7 \mathrm{~m} \mathrm{~s}^{-1}$ \\
$\mathrm{~A}\left(<75 \mathrm{~m} \mathrm{~s}^{-1}\right)$ & North & $0.21 \pm 0.04$ & 43.2 & $262.8 \mathrm{~m} \mathrm{~s}^{-1}$ \\
\hline
\end{tabular}

\subsection{Results for TIGER radar $v_{\text {LOS }}$ (electric field)}

Figure 13 shows the PDFs of $v_{\text {LOS }}$ for $\tau=1,2,4, \ldots$, 256 min observed using the TIGER radar in the nightside high-latitude ionosphere. Part (a) shows the results for Population $\mathrm{B}$ (spectral width $\geq 150 \mathrm{~m} \mathrm{~s}^{-1}$ ), and part (b) shows the results for Population A (spectral width $\leq 75 \mathrm{~m} \mathrm{~s}^{-1}$ ). As explained in Sect. 3.1, Population B echoes are more likely to be found on open field lines in the polar cap, but also on closed field lines just equatorward of the open-closed boundary, whereas Population A echoes are more likely to be found on closed field lines in the auroral and sub-auroral ionosphere. Population A echoes are also more likely to be found in regions of high Pedersen conductance which often corresponds to the auroral ionosphere (Parkinson et al., 2004).

The PDFs of $v_{\text {LOS }}$ for Population B (Fig. 13a) are highly skewed toward positive values, and they appear single peaked. The positive skew occurs because the distribution is dominated by anti-sunward flows exiting the polar cap (cf. Fig. 7). Not surprisingly, the PDFs shift more (less) toward positive values for $B_{z}$ southward (northward). The PDFs appear single peaked, but they may actually be the synthesis of three overlapping but unresolved distributions. There are negative (sunward) flows, and close examination reveals a small enhancement near $v_{\mathrm{LOS}}=0 \mathrm{~m} \mathrm{~s}^{-1}$, possibly caused by ground scatter mistaken for ionospheric scatter.

The PDFs of $v_{\text {LOS }}$ for Population A (Fig. 13b) are trimodal, consisting of a dominate peak associated with antisunward flows exiting the polar cap (motion towards the radar), a secondary peak centred close to $v_{\mathrm{LOS}}=0 \mathrm{~m} \mathrm{~s}^{-1}$, and a tertiary peak associated with sunward flows. Because of expansion and contraction of the auroral oval, the ratio of the amplitude of positive $v_{\text {LOS }}$ peak to the negative $v_{\text {LOS }}$ peak increases (decreases) for $B_{z}$ southward (northward). The secondary peak is mostly due to zonal flows in the auroral and sub-auroral ionosphere. It becomes the dominate peak when $B_{z}$ is northward, and the tertiary peak when $B_{z}$ is southward. These changes are also consistent with the expected expansion and contraction of the polar cap with $B_{z}$. 


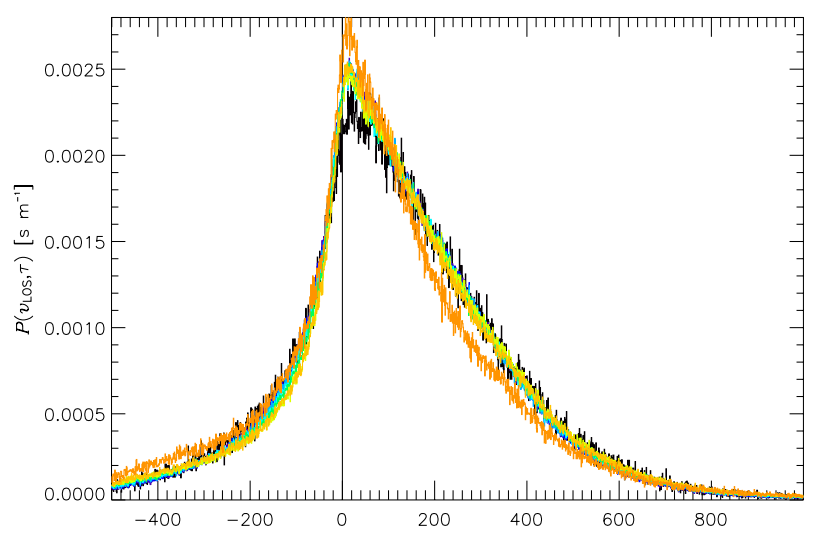

(a)

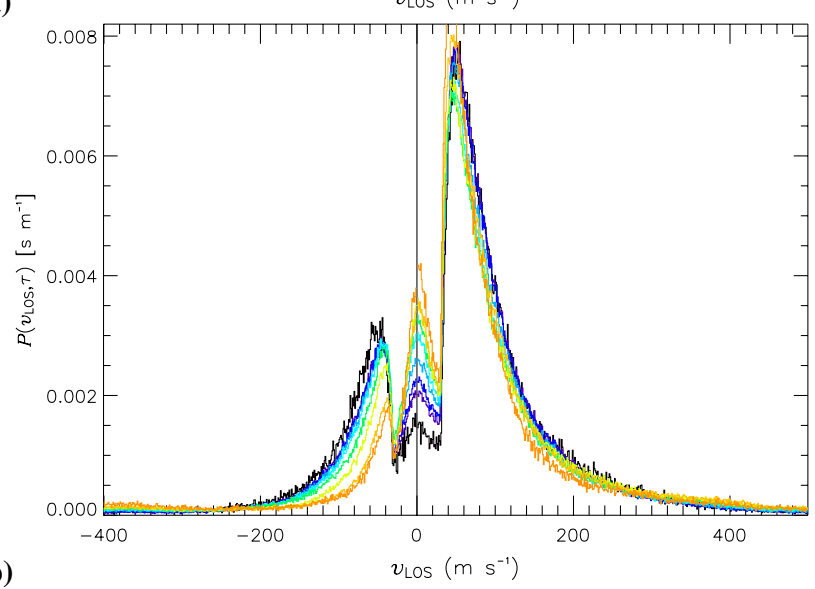

(b)

Fig. 13. PDFs of $v_{\mathrm{LOS}}$ for $\tau=1 \mathrm{~min}$ (black), $2 \mathrm{~min}, \ldots, 256 \mathrm{~min}$ (orange) observed using the TIGER radar during 20:00 to 02:00 MLT. The results have been sorted according to (a) spectral width $\geq 150 \mathrm{~m}$ $\mathrm{s}^{-1}$ (Population B), and (b) spectral width $\leq 75 \mathrm{~m} \mathrm{~s}^{-1}$ (Population A). The number of samples varied from 266388 for $\tau=1 \mathrm{~min}$ to 1625514 for $\tau=16 \mathrm{~min}$ for Population B, and from 117421 for $\tau=1 \mathrm{~min}$ to 591619 for $\tau=2 \mathrm{~min}$ for Population A. A bin size of $1 \mathrm{~m}$ $\mathrm{s}^{-1}$ was used for $v_{\mathrm{LOS}}$.

Figure 13a shows that some large positive $v_{\mathrm{LOS}}$ values are less likely to occur at $\tau=256 \mathrm{~min}$ (orange), and Fig. 13b shows that the secondary peak close to $v_{\mathrm{LOS}}=0 \mathrm{~m} \mathrm{~s}^{-1}$ grows in amplitude with $\tau$. These effects occur because the $v_{\text {LOS }}$ samples at $\tau=1$ min were measured during 20:01 to 02:00 MLT, whereas at $\tau=256 \mathrm{~min}$ they were measured during 00:44 to 02:00 MLT (i.e. 256 min after 20:00 MLT). The convection pattern tends to become more zonal when the radar FoV is in the latter MLT sector.

The same procedures used to estimate scaling parameters for the Halley radar data in preceding section were applied to the TIGER data. Table 1 lists the values of $\alpha_{0}, \alpha_{\mathrm{GSF}}, H$, and their errors for Populations A and B, and Table 3 lists the values of $\alpha_{0}$ for $B_{z}$ southward and northward conditions. In most cases, the individual data points were reasonably close to the best fit straight line, but as previously explained, the largest errors occurred for $\tau=1$ and $256 \mathrm{~min}$. Kurtosis and

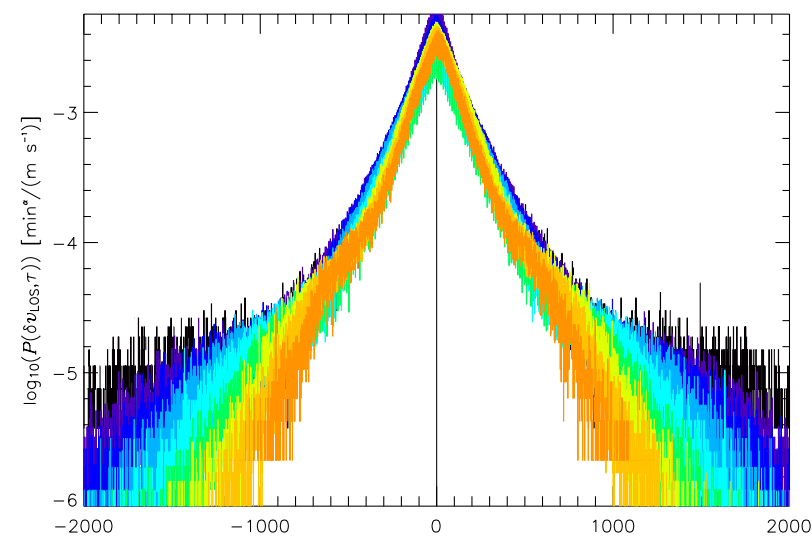

(a)

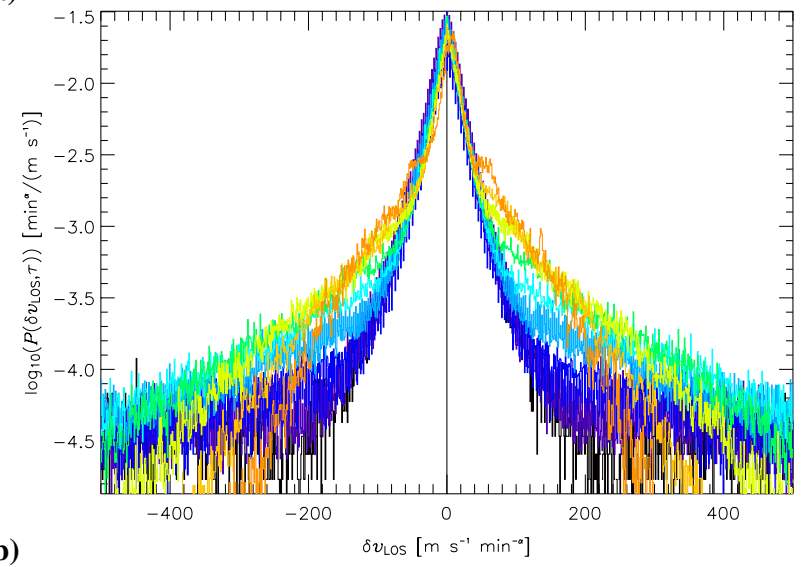

Fig. 14. The extent to which the PDFs of $\delta v_{\text {LOS }}$ collapse to a common curve for (a) TIGER Population B, and (b) TIGER Population A. Common bin sizes of $1 \mathrm{~m} \mathrm{~s}^{-1}$ were used for $\delta v_{\text {LOS }}$ prior to scaling.

RMS values of un-scaled $v_{\text {LOS }}$ values averaged over $\tau=1$ to $256 \mathrm{~min}$ are also given.

Figure 14a shows the extent to which the PDFs of $\delta v_{\text {LOS }}$ collapse to the same curve for TIGER Population B. The scaling collapse of the PDFs was satisfactory out to $\delta v_{\mathrm{LOS}} \approx \pm 800 \mathrm{~m} \mathrm{~s}^{-1}$ for all $\tau$, but the probability of $\left|\delta v_{\mathrm{LOS}}\right|>800 \mathrm{~m} \mathrm{~s}^{-1}$ decreases with $\tau$. That is, the same "role off" of the wings found for Halley Population B (cf. Fig. 12a) is steeper and more clearly defined for TIGER echoes because of the greater number of available samples. The effect was strongest for $\tau=256 \mathrm{~min}$ because the mapping $\delta v_{\mathrm{LOS} s}=\delta v_{\mathrm{LOS}} \tau^{-\alpha_{0}}$ implies the role off corresponds to measured velocities of $\sim 2.6 \mathrm{~km} \mathrm{~s}^{-1}$, the Nyquist limit of the radar. Strong leptokurtic wings persisted for $\tau=1 \mathrm{~min}$, as they might for larger $\tau$ if the radars could measure velocities in the order of $10 \mathrm{~km} \mathrm{~s}^{-1}$.

Figure 14b shows that mono-scaling collapse of the PDFs for Population A was not achieved. Here we used $\alpha_{0}=0.35 \pm 0.04$, but varying this parameter did not improve the scaling significantly. Whilst the PDFs collapsed to the same curve out to $\delta v_{\mathrm{LOS}} \approx \pm 40 \mathrm{~m} \mathrm{~s}^{-1}$, even this result is 
questionable because measurement errors most strongly affected the peaks of the PDFs (i.e. Fig. 9). We conclude the results for TIGER Population A are not self-affine, even less self-affine than for Halley Population B. When allowing for errors, the graph of $\zeta(m)$ versus $m$ suggests the results might be multi-fractal.

When the PDFs for Population A and B were sorted according to the IMF, large values of $\delta v_{\mathrm{LOS}}$ had a greater probability for $B_{z}$ northward. Only minor changes were found when the PDFs were sorted according to $B_{y}$.

\section{Discussion}

An ongoing concern in the field of magnetospheric complexity science is the extent to which the magnetosphere is a SOC system, or a forced SOC system (F/SOC) (Chang, 1992). Some aspects of magnetospheric dynamics are directly driven by the solar wind, yet others seem to arise from internal interactions, and it is possible the dynamics are often driven yet self-organised, behaving in a way analogous to phase transitions (Ukhorskiy et al., 2003). The driver might dominate the magnetospheric dynamics when and where the coupling is strong, yet self-organisation might prevail when and where the coupling is weak. For example, it is well known that fluctuations in the dayside cusp currents and electric fields in the ionosphere respond rapidly to changing solar wind conditions. The $D P 2$ current system and related electric fields might also fluctuate in a way that is partly determined by the driver.

Although the overall shape of the magnetotail owes its very existence to the solar wind, much of its complicated internal structure might be self-organised. Many $D P 1$ substorm current un-loadings are triggered by IMF northward turnings, but there are so-called spontaneous substorms (Borovsky, 1993). Also, there is a distinction between the driver and trigger for a system near to criticality. For example, the weather ultimately drives snow avalanches, yet the trigger may be any perturbation, such as a skier traversing the slope. In the case of substorms, an external trigger does not preclude self-organisation. Given the spontaneity of substorms, it seems reasonable to suggest that much of the internal structure and evolution of the magnetotail is not directly driven.

Our re-scaled radar observations are in basic agreement with this broad picture of the magnetosphere. Halley Population B corresponds to echoes from the dayside greater cusp, and mostly included the effects of electric field fluctuations associated with the $D P 2$ and cusp current systems. Like the solar wind $\varepsilon$ fluctuations (Fig. 3), the Halley Population B results exhibited the most convincing mono-fractal scaling (Fig. 12a). Halley Population A results also exhibited reasonable self-affinity (Fig. 12b). Although these low spectral width echoes are thought to map to closed field lines (Baker et al., 1995), they include echoes which map to the low-
Table 3. Scaling parameters $\alpha_{0}$ for $v_{\text {LOS }}$ observed by the TIGER radar in the nightside ionosphere.

\begin{tabular}{llcrl}
\hline $\begin{array}{l}\text { Population } \\
\text { (spectral width) }\end{array}$ & $B_{z}$ & $\alpha_{0}$ & $<\kappa>$ & $<\delta v_{\text {RMS }}>$ \\
\hline $\mathrm{B}\left(>150 \mathrm{~m} \mathrm{~s}^{-1}\right)$ & All & $0.18 \pm 0.04$ & 9.9 & $402.2 \mathrm{~m} \mathrm{~s}^{-1}$ \\
$\mathrm{~B}\left(>150 \mathrm{~m} \mathrm{~s}^{-1}\right)$ & South & $0.18 \pm 0.04$ & 10.3 & $397.8 \mathrm{~m} \mathrm{~s}^{-1}$ \\
$\mathrm{~B}\left(>150 \mathrm{~m} \mathrm{~s}^{-1}\right)$ & North & $0.19 \pm 0.04$ & 9.5 & $404.5 \mathrm{~m} \mathrm{~s}^{-1}$ \\
$\mathrm{~A}\left(<75 \mathrm{~m} \mathrm{~s}^{-1}\right)$ & All & $0.35 \pm 0.04$ & 22.6 & $379.6 \mathrm{~m} \mathrm{~s}^{-1}$ \\
$\mathrm{~A}\left(<75 \mathrm{~m} \mathrm{~s}^{-1}\right)$ & South & $0.34 \pm 0.04$ & 34.4 & $306.0 \mathrm{~m} \mathrm{~s}^{-1}$ \\
$\mathrm{~A}\left(<75 \mathrm{~m} \mathrm{~s}^{-1}\right)$ & North & $0.35 \pm 0.04$ & 14.5 & $459.0 \mathrm{~m} \mathrm{~s}^{-1}$ \\
\hline
\end{tabular}

latitude boundary layer, a region exhibiting dynamics closely related to the cusp, and perhaps sometimes residing on open field lines. Because of the conducting, incompressible ionosphere, fluctuations in the electric potential generated by reconnection across the merging gap may penetrate to closed field lines laying just equatorward of the open-closed magnetic field line boundary.

TIGER Population B echoes also exhibited good selfaffinity (Fig. 14a). Within experimental error, they shared the same scaling parameters as Halley Population B (Table 1). However, the fluctuations levels, as measured by $\left\langle\delta v_{\mathrm{RMS}}>\right.$, were slightly larger for Halley Population B. Most TIGER Population B echoes map to open magnetic field lines in the tail lobes, and they must be associated with the $D P 2$ current system, and partly the $D P l$ current system. TIGER Population A echoes are found further equatorward and they map to the plasma sheet in the magnetotail, a region thought to exhibit multi-fractal behaviour (Lui, 2001). Our TIGER Population A echoes did not exhibit mono-fractal scaling (Fig. 14b). These fluctuations must include strong effects associated with substorm DPl current un-loadings which might constitute system wide avalanches. In the future, we aim to further sort all the PDFs according to substorm phase $(D P 1)$ and non-substorm (DP2) conditions, as well as perform a multi-fractal analysis.

The PDFs of $\delta v_{\text {LOS }}$ probably collapsed to the same curve for Halley and TIGER Populations B for $\tau=1$ to $256 \mathrm{~min}$, but less so for Halley Population A. The existence of this power law scaling is an attribute of SOC, but some other, perhaps unknown linear or non-linear dynamic might also explain our results. Nor do our results prove the fluctuating ionospheric electric fields are directly driven by corresponding fluctuations in the solar wind. Coincidentally, both space plasmas may be governed by nonlinear dynamics exhibiting similar scaling. The importance of the solar wind driver might be revealed by a multi-year study of seasonal and solar cycle variations in the scaling parameters, revealing common systematic variations.

SuperDARN radars were designed to measure convection speeds in the order of $\sim 100 \mathrm{~m} \mathrm{~s}^{-1}$ to $2-3 \mathrm{~km} \mathrm{~s}^{-1}$, and they 
provide an outstanding view of global-scale electrodynamics (Ruohoniemi and Baker, 1998). The invention of the pulse set/FITACF approach achieved a large Nyquist frequency whilst reducing the deleterious affects of range aliasing when sampling out to great ranges (1000 to $3000 \mathrm{~km})$. However, the radar data has its limitations and our interpretation took them into account. Of fundamental concern is the ability of existing radars to measure very small $\left(\sim 1-10 \mathrm{~m} \mathrm{~s}^{-1}\right)$ and very large $\left(>2 \mathrm{~km} \mathrm{~s}^{-1}\right)$ velocity with small relative error. The shapes of the PDFs of $\delta v_{\text {LOS }}$ are probably reasonable for intermediate velocities, but the peaks of the PDFs might become sharper with increased Doppler resolution. Also, the shapes of the present PDFs imply the probability density in the long tails would increase with improved angular and range resolution, and Nyquist frequency. The PDFs may be even more leptokurtic than shown (cf. Fig. 9). This implies the existence of intense flows at small scale sizes.

Lanchester et al. (1996) reported high space and time (9s) resolution EISCAT measurements of electric field coincident with TV images of optical aurora. These measurements revealed the existence of intense $\left(\sim 400 \mathrm{mV} \mathrm{m}^{-1}\right)$, short-lived $(\sim$ minute) electric fields occurring at the edges of auroral arcs. Other TV images of optical aurora reveal intense, small-scale $(<10 \mathrm{~km})$ filamentary currents with lifetimes $<1 \mathrm{~s}$, and no doubt these are also accompanied by intense electric fields. The fractal limits of filamentary current and electric field structure is unknown, but we expect it to extend down to the same scale size of ionospheric irregularities and energy dependent gyro-radii $(<1 \mathrm{~m})$. Although the radars do not access the regime of rare, intense, and spatially localised fluctuations of most relevance to magnetospheric complexity, the long tails of the PDFs of $\delta v_{\text {LOS }}$ do imply the existence of such fluctuations. Combined, these results imply the existence of convection "microbursts". Radars with the spatial and temporal resolution required to analyse this regime need to be developed.

Further experimental support for the relevance of intense small-scale electric field fluctuations is provided by an intermittency analysis of high time resolution SIERRA rocket measurements (Tam et al., 2005). They identified a power law scaling regime for fluctuations in auroral electric fields extending from $5 \mathrm{~ms}$ to $\sim 1 / 3 \mathrm{~s}$ in the rocket reference frame. The smallest scale fluctuations exhibit the strongest intermittency. The treatise of Chang et al. (2004) provides theoretical support for the cause of intermittent plasma turbulence at these small scales, and hence the cause of complexity in space plasmas. These recent studies also suggest that improved HF radars would measure intense activity at smaller scales.

It was not possible to reliably infer the outer limit of the scaling regime because the Nyquist frequency of $\sim 2.6 \mathrm{~km}$ $\mathrm{s}^{-1}$ caused a steep role off in the re-scaled PDFs at the largest $\tau$. Also, at the longest time scales, $\tau \geq 256 \mathrm{~min}$, the $\delta v_{\text {LOS }}$ values became a mixture of spatial and temporal fluctuations. We attributed some of the discrepancies in the PDFs to aver- age spatial variations in the underling statistical convection pattern. Although this is a second-order effect, ideally we require a radar which resides in the same MLT sector as the Earth rotates. However, given the earlier Hnat et al. (2002b, 2003a) results obtained using the AE indices, the present results confirmed the outer limit of the scaling regime extended to near $\tau=128$ or $256 \mathrm{~min}$.

TIGER Population A corresponds to an ionospheric regime dominated by energetic particle precipitation and suppression of electric field fluctuations by large Pedersen conductance (Parkinson et al., 2004). It is well known that magnetic field lines are not equipotentials in the auroral ionosphere because of field-aligned potential drops. Large-scale electric fields map from the magnetosphere to the ionosphere in the auroral oval, but the short-scale electric fields are suppressed (Weimer et al., 1985). Hence the "Pedersen conductance filter" will have the largest impact at the shortest time scales for TIGER Population A. Ideally, the transfer function specifying how fluctuations in the magnetosphere map to the ionosphere ought to be measured and de-convolved from the radar measurements.

Perhaps it is surprising that scaling collapse was achieved for all of the PDFs from $\tau=1$ to 256 min because the chosen values of $\tau$ crossed the time scales of different physical processes. Solar wind fluctuations and the magnetospheric interchange instability probably drive electric field fluctuations across a broad range of time scales. ULF waves contribute to the fluctuation level for $\tau<15 \mathrm{~min}$, whereas atmospheric gravity waves might contribute for $15 \mathrm{~min}<\tau<120 \mathrm{~min}$, and tides and planetary waves at even longer $\tau$. However, the fact that scaling collapse was achieved suggest the role of ULF waves was secondary, or they shared the same stochastic properties of the observed fluctuating electric fields. Gravity waves might be generated with periods similar to the electric field fluctuations driving them via the Lorentz force, whereas the effects of tides and planetary waves occur at longer time scales than analysed here.

Golovchanskaya et al. (2002) used Dynamics Explorer 2 (DE 2) observations to show the high-latitude convection is more likely to be smooth when it is strongly driven under $B_{z}$ southward conditions, and that electric field fluctuations increase in intensity on closed field lines under $B_{z}$ northward conditions. They propose that when $B_{z}$ is northward, interchange instability develops within closed field line regions mapping to high latitude. They also reported their DE 2 electric field fluctuations had a most common scale size of $11 \mathrm{~km}$, an irregularity scale size comparable to the smallscale vortices thought to strongly influence the Doppler spectral widths measured by SuperDARN radars (Huber and Sofko, 2000).

Similarly, the present HF radar observations show evidence for a $B_{z}$ northward enhancement of fluctuation level. Tables 2 and 3, show that $<\delta v_{\text {RMS }}>$ was larger for both Halley and TIGER Populations A when $B_{z}$ was northward, but for neither Population B. Population A echoes tend to occur 
on closed field lines mapping to deeper inside the magnetosphere where interchange instability will enhance $\delta v_{\mathrm{LOS}}$, whereas Population B echoes occur at higher latitude on open field lines, and on closed field lines just equatorward of the open-closed boundary.

In conceptual models of magnetosphere-ionosphere coupling, the open-closed magnetic field line boundary is adiaroic and represented by a smooth line (fractal dimension = 1). However, the present results show the convective flow has a large fractal dimension, especially in proximity to the dayside cusp. This raises the possibility that an adiaroic openclosed boundary is also fractal. The radar spectral width boundary, often taken as a proxy for the open-closed boundary, exhibits structure down to the time and range resolution of the measurements (see Parkinson et al., 2002). The iso-contours of auroral intensity are also known to be fractal (e.g., Kozelov, 2003). The fractal dimension of the poleward boundary of the auroral oval, and other proxies for the openclosed boundary, need to be determined.

\section{Summary}

1. The solar wind $\varepsilon$ parameter was estimated using ACE spacecraft measurements during year 2000. As with previous studies, the PDFs of fluctuations in $\varepsilon$ at various time scales $\tau$ were compiled and found to be leptokurtic. There was a greater occurrence of small (large) $\delta \varepsilon$ values for small (large) values of $\tau$, with the cross over points located near $\delta \varepsilon \sim \pm 0.3^{10} \mathrm{~W}$.

2. Peaks in the PDFs were used to estimate the scaling parameter $\alpha_{0}$. Estimates of $\alpha_{0}$ were vexed because of the $\sin ^{4}(\theta / 2)$ term in the definition of $\varepsilon$, combined with sensitivity to measurement errors. $\alpha_{0}$ increased with decreasing bin size used to resolve the peaks but there was a local maximum of $\alpha_{0}=0.43 \pm 0.03$ for $\Delta \delta \varepsilon \sim 0.00001^{10} \mathrm{~W}$, in agreement with earlier work.

3. GSF analysis was used to estimate the scaling parameter $\alpha_{\mathrm{GSF}}=0.25 \pm 0.05$ and the Hurst exponent $H=0.23 \pm 0.05$. The moments of the GSF analysis of $\delta \varepsilon$ exhibited power law scaling over 9 octaves of time scale ranging from $46 \mathrm{~s}$ to $\sim 9.1 \mathrm{~h}$, and scaling collapse of the PDFs was also achieved. The $\delta \varepsilon$ fluctuations were self-affine (mono-fractal) over this regime.

4. SuperDARN radar measurements of ionospheric $v_{\text {LOS }}$ in the meridional direction (equivalent to zonal electric field) were analysed in the same way as the $\varepsilon$ parameter. The HF radar measurements were sorted according to Halley measurements in the dayside ionosphere (08:00 to 14:00 MLT) and TIGER measurements in the nightside ionosphere (20:00 to 02:00 MLT). The analyses were further sorted according to regions of high spectral width (Population B, $>150 \mathrm{~m} \mathrm{~s}^{-1}$ ) occurring predominantly on open field lines within the polar cap, and low spectral width (Population A, $<75 \mathrm{~m} \mathrm{~s}^{-1}$ ) occurring predominantly on closed field lines mapping deeper inside the magnetosphere. The analysis was also sorted according to basic categories of the IMF.

5. The PDFs of $v_{\text {LOS }}$ measured by both radars were in basic agreement with standard two-cell convection patterns, and they also changed in shape with $B_{z}$ in the expected way. Large $v_{\text {LOS }}$ values occurred most often for Halley Population $\mathrm{B}$ in the greater cusp, and small $v_{\mathrm{LOS}}$ values occurred most often for TIGER Population A mapping to the nightside inner magnetosphere.

6. PDFs of fluctuations in $v_{\text {LOS }}$ were compiled for time scales $\tau$ from $1 \mathrm{~min}$ to $256 \mathrm{~min}$. The smallest time scale was limited by the time resolution of the radar scans and the largest time scale by rotation of the radars in MLT. The PDFs of $\delta v_{\text {LOS }}$ were leptokurtic and modeling of the weakly correlated velocity errors suggested the leptokurtic peaks would "sharpen" if the errors were reduced. The effective Nyquist frequency of the measurements limited the probability density in the long tails of the leptokurtic functions.

7. Peaks in the PDFs of $\delta v_{\text {LOS }}$ and GSF analyses were used to estimate scaling parameters for the radar measurements. The scaling parameters were then used to test for scaling collapse. Halley Population B exhibited the most convincing self-affinity (like the $\varepsilon$ parameter) out to the Nyquist limit. Halley Population A and TIGER Population B also exhibited self-affinity, whereas TIGER Population A did not exhibit scaling collapse. Due to the conducting, incompressible ionosphere, fluctuations in the electric potential in the open magnetosphere (Halley Population B) can penetrate to the closed field lines residing just equatorward of the openclosed boundary (Halley Population A). Thus the three most outer domains (Halley Populations B and A, and TIGER Population B) exhibit scaling most akin to the solar wind, whereas TIGER Population A tends to map to the nightside plasma sheet, a separate electrodynamic region generating DP1 substorm current unloadings.

8. Referring to Tables 1 to 3, the scaling parameters obtained for Halley and TIGER Population B were essentially the same, namely $\alpha_{0}=0.185 \pm 0.04$. The zonal electric field fluctuations exhibited the strongest mono-fractal cascade in these higher latitude regions. For the dayside Halley Population $\mathrm{A}, \alpha_{0}$ was $0.23 \pm 0.04$, the next most fractal region. For the nightside TIGER Population A, $\alpha_{0}$ was $0.35 \pm 0.04$, but scaling collapse did not occur. Electrodynamic fluctuations in the nightside plasma sheet are known to be multi-fractal. No significant variations in the scaling parameters were detected between $B_{z}$ north and south categories for any of the morphological regions, suggesting an internal organisation of fluctuation.

9. Finally, referring to Tables 2 and 3, the average RMS velocity fluctuations, $<\delta v_{\mathrm{RMS}}>$, were largest for Halley Population B, next largest for TIGER Population B, and weakest for Halley Population A. The $<\delta v_{\mathrm{RMS}}>$ values were substantially smaller (larger) for $B_{z}$ southward (northward) for both Halley and TIGER Population A which tend to map to 
closed field lines in the inner magnetosphere. Again, this is further evidence for an internal organisation of fluctuation.

An important consequence of this work is that any dynamical model predicting variability in magnetosphereionosphere behaviour on time scales $<4 \mathrm{~h}$ should exhibit statistical scaling properties like those found in this study, otherwise the predictions may be flawed in some way.

Acknowledgements. This work was supported by the Australian Research Council, the Australian Antarctic Science Advisory Committee, and the Australian Academy of Sciences. N. Watkins, B. Hnat, S. Chapman, G. Abel, and M. Freeman are thanked for insightful discussions. D. J. McComas of the Los Alamos National Laboratory and N. F. Ness of the Bartol Research Institute are thanked for making ACE solar wind and magnetic field data available, respectively. All of those who contributed to the construction, maintenance, and operation of the Halley and TIGER SuperDARN radars are also thanked.

Topical Editor T. Pulkkinen thanks A. Kozlovsky and another referee for their help in evaluating this paper.

\section{References}

Abel, G. A. and Freeman, M. P.: A statistical analysis of ionospheric velocity and magnetic field power spectra at the time of pulsed ionospheric flows, J. Geophys. Res., 107(A12), 1470, doi:10.1029/2002JA009402, 2002.

Bak, P., Tang, C., and Wiesenfeld, K.: Self-organized criticality: An explanation of 1/f noise, Phys. Rev. Lett., 59(4), 381-384, 1987.

Baker, K. B. and Wing, S.: A new magnetic coordinate system for conjugate studies of high latitudes, J. Geophys. Res., 94, 91399143, 1989.

Baker, K. B., Dudeney, J. R., Greenwald, R. A., et al.: HF radar signatures of the cusp and low-latitude boundary layer, J. Geophys. Res., 100, 7671-7695, 1995.

Baker, D. N., Kanekal, S. G., Klimas, A. J., and Vassilliadis, D.: Collective phenomena in the inner magnetosphere, Phys. Plasmas, 6(11), 4195-4199, 1999.

Borovsky, J. E., Nemzek, R. J., and Belian, R. D.: The occurrence rate of magnetospheric substorm onsets: randomn and periodic substorms, J. Geophys. Res., 98, 3807-3813, 1993.

Castaing, B., Gagne, Y., and Hopfinger, E. J.: Velocity probability density functions of high Reynolds number turbulence, Physica D, 46, 177-200, 1990.

Chang, T. S.: Low-dimensional behaviour and symmetry breaking of stochastic systems near criticality - can these effects be observed in space and in the laboratory?, IEEE Trans. Plasma Sci., 20, 691-694, 1992.

Chang, T., Tam, S. W. Y., and Wu, C.-C.: Complexity induced anisotropic bimodal intermittent turbulence in space plasmas, Phys. of Plasmas, 11, 1287-1299, 2004.

Chisham, G., Freeman, M. P., and Sotirelis, T.: A statistical comparison of SuperDARN spectral width boundaries and DMSP particle precipitation boundaries in the nightside ionosphere, Geophys. Res. Lett., 31, L02804, doi:10.1029/2003GL019074, 2004.

Chisham, G., Freeman, M. P., Sotirelis, T., Greenwald, R. A., Lester, M., and Villain, J.-P.: A statistical comparison of SuperDARN spectral width boundaries and DMSP particle precip- itation boundaries in the morning sector ionosphere, Ann. Geophys., 23, 733-743, 2005.

Consolini, G. and Chang, T. S.: Magnetic field topology and criticality in geotail dynamics: relevance to substorm phenomena, Space Sci. Rev., 95, 309-321, 2001

Dudeney, J. R., Rodger, A. S., Freeman, M. P., Pickett, J., Scudder, J., Sofko, G., and Lester, M.: The nightside ionospheric response to IMF B $y$ changes, Geophys. Res. Lett., 25, 2601-2604, 1998.

Dyson, P. L. and Devlin, J. C.: The Tasman International Geospace Environment Radar, The Physicist (The Australian Institute of Physics), 37, 48-53, March/April, 2000.

Dyson, P. L., Devlin, J. C., Parkinson, M. L., and Whittington, J. S.: The Tasman International Geospace Environment Radar (TIGER) - Current Development and Future Plans, IEEE Proceedings of the International Conference on Radar, IEEE Catalogue No. 03EX695C, 282-287, 2003.

Freeman, M. P., Watkins, N. W., and Riley, D. J.: Evidence for a solar wind origin of the power law burst lifetime distribution of the AE indices, Geophys. Res. Lett., 27(8), 1087-1090, 2000.

Frette, V., Christensen, K., Malthesorenssen, A., Feder, J., Jossang, T., and Meakin, P.: Avalanche dynamics in a pile of rice, Nature, 379(6560), 49-52, 1996

Golovchanskaya, I. V., Maltsev, Y. P., and Ostapenko, A. A.: Highlatitude irregularities of the magnetospheric electric field and their relation to solar wind and geomagnetic conditions, J. Geophys. Res., 107(A1), doi:10.1029/2001JA900097, 2002.

Greenwald, R. A., Baker, K. B., Dudeney, J. R., et al.: DARN/SuperDARN: A global view of the dynamics of highlatitude convection, Space Sci. Rev., 71, 761-796, 1995.

Huber M. and Sofko G. J.: Small-scale vortices in the high-latitude F region, J. Geophys. Res., 105(A9), 20 885-20 897, 2000.

Hnat, B., Chapman, S. C., Rowlands, G., Watkins, N. W., and Farrell, W. M.: Finite size scaling in the solar wind magnetic field energy density as seen by WIND, Geophys. Res. Lett., 29(10), doi:10.1029/2001GL014587, 2002a.

Hnat, B., Chapman, S. C., Rowlands, G., Watkins, N. W., and Freeman, M. P.: Scaling of the solar wind $\varepsilon$ and the $\mathrm{AU}, \mathrm{AL}$ and $\mathrm{AE}$ indices as seen by WIND, Geophys. Res. Lett., 29(22), 2078, doi:10.1029/2002GL016054, 2002b.

Hnat, B., Chapman, S. C., Rowlands, G., Watkins, N. W., and Freeman, M. P.: Scaling in long term data sets of geomagnetic indices and solar wind $\varepsilon$ as seen by WIND spacecraft, Geophys. Res. Lett., 30(22), 2174, doi:10.1029/2003GL018209, $2003 \mathrm{a}$.

Hnat, B., Chapman, S. C., and Rowlands, G.: Intermittency, scaling, and the Fokker-Planck approach to fluctuations of the solar wind bulk plasma parameters as seen by the WIND spacecraft, Phys. Rev. E, 67(5), 56404-1-8, 2003b.

Hnat, B., Chapman, S. C., and Rowlands, G.: Scaling and a FokkerPlank model for fluctuations in geomagnetic indices and comparison with solar wind $\varepsilon$ as seen by WIND and ACE, J. Geophys. Res., 110, A08206, doi:10.1029/2004JA010824, 2005.

Janicki, A., Popova, I., Ritchken, P. H., and Woyczynski, W. A.: Option pricing bounds for $\alpha$-stable stochastic models, Mathematics \& Computers in Simulation, 2005.

Jensen, H. J.: Self-organised criticality: Emergent complex behaviour in physical and biological systems, Cambridge University Press, Cambridge, 1998.

Kelley, M. C.: The Earth's Ionosphere, Plasma Physics and Electrodynamics, Academic Press, London, 1989. 
Klimas, A. J., Valdivia, J. A., Vassiliadis, D., Baker, D. N., Hesse, M., and Takalo, J.: Self-organized criticality in the substorm phenomenon and its relation to localized reconnection in the magnetospheric plasma sheet, J. Geophys. Res., 105(A8), 18765 $18780,2000$.

Kozelov, B. V.: Fractal approach to description of auroral structure, Ann. Geophys., 21, 2011-2023, 2003.

Lanchester, B. S., Kaila, K., and McCrea, I. W.: Relationship between large horizontal electric fields and auroral arc elements, J. Geophys. Res., 101(A3), 5075-5084, 1996.

Lester, M., Milan, S. E., Besser, S. E., and Smith, R.: A case study of $\mathrm{HF}$ radar spectra and $630.0 \mathrm{~nm}$ auroral emission in the premidnight sector, Ann. Geophys., 19, 327-339, 2001.

Lui, A. T. Y.: Multifractal and intermittent nature of substormassociated magnetic turbulence in the magnetotail, J. Atmos. Solar-Terr. Phys., 63, 1379-1385, 2001.

Lui, A. T. Y., Chapman, S. C., Liou, K., et al.: Is the dynamic magnetosphere an avalanching system?, Geophys. Res. Lett., 27(7), 911-914, 2000

Lu, E. T.: Avalanches in continuum driven dissipative systems, Phys. Rev. Lett., 74(13), 2511-2514, 1995.

Mandelbrot, B. B.: Gaussian self-affinity and fractals: Globality, the Earth, 1/f noise and R/S, Springer-Verlag, Berlin, 2002.

Mantegna, R. N. and Stanley, H. E.: Scaling behaviour in the dynamics of an economic index, Nature, 376, 46-49, 1995.

McComas, D. J., Bame, S. J., Barker, P., Feldman, W. C., Phillips, J. L., Riley, P., and Griffee, J. W.: Solar wind electron proton alpha monitor (SWEPAM) for the Advanced Composition Explorer, Space Sci. Rev., 86, 563-612, 1998.

Pagel, C. and Balogh, A.: Intermittency in the solar wind: A comparison between solar minimum and maximum using Ulysses data, J. Geophys. Res., 107(A8), 1178, doi:10.1029/2002JA009331, 2002.

Palmer, S.: Snow science, Focus, 77-84, January, 2003.

Papitashvili, V. O. and Rich, F. J.: High-latitude ionospheric convection models derived from Defense Meteorological Satellite Program ion drift observations and parameterized by the interplanetary magnetic field strength and direction, J. Geophys. Res., 107(A8), doi:10.1029/2001JA000264, 2002.

Parkinson, M. L., Breed, A. M., Dyson, P. L., and Morris, R. J.: Signatures of the ionospheric cusp in digital ionosonde measurements of plasma drift above Casey, Antarctica, J. Geophys. Res., 104(A10), 22 487-22 498, 1999.

Parkinson, M. L., Dyson, P. L., Pinnock, M., Devlin, J. C., Hairston, M. R., Yizengaw, E., and Wilkinson, P. J.: Signatures of the midnight open-closed magnetic field-line boundary during balanced dayside and nightside reconnection, Ann. Geophys., 20, 16171630, 2002.

Parkinson, M. L., Devlin, J. C., Ye, H., Waters, C. L., Dyson, P. L., Breed, A. M., and Morris, R. J.: On the occurrence and motion of decametre-scale irregularities in the sub-auroral, auroral, and polar cap ionosphere, Ann. Geophys., 21, 1847-1868, 2003.
Parkinson, M. L., Chisham, G., Pinnock, M., Dyson, P. L., and Devlin, J. C.: Magnetic local time, substorm, and particle precipitation-related variations in the behaviour of SuperDARN Doppler spectral widths, Ann. Geophys., 22, 4103-4122, 2004.

Pinnock, M., Rodger, A. S., Dudeney, J. R., Baker, K. B., Newell, P T., Greenwald, R. A., and Greenspan, M. E.: Observations of an enhanced convection channel in the cusp ionosphere, J. Geophys. Res., 98, 3767-3776, 1993.

Ruohoniemi, J. M. and Baker, K. B.: Large-scale imaging of highlatitude convection with Super Dual Auroral Radar Network HF radar observations, J. Geophys. Res., 103, 20 797-20 811, 1998.

Ruohoniemi, S. G., Shepherd, J. M., and Greenwald, R. A.: The response of the high-latitude ionosphere to IMF variations, J. Atmos. Solar-Terr. Phys., 64, 159-171, 2002.

Smith, C. W., Acuña, M. H., Burlaga, M. F., L'Heureux, J., Ness, N. F., and Scheifele, J.: The ACE Magnetic Fields Experiment, Space Sci. Rev., 86, 613-632, 1998.

Sornette, D.: Critical phenomena in natural sciences; chaos, fractals, selforganization, and disorder; concepts and tools, SpringerVerlag, Berlin, 2000.

Spiegel, M. R. and Boxer, R. W.: Theory and Problems of Statistics, Schaum's Outline Series, McGraw-Hill, New York, 1972.

Tam, S. W., Chang, T., Kintner, P. M., and Klatt, E.: Intermittency analyses on the SIERRA measurements of the electric field fluctuations in the auroral zone, Geophys. Res. Lett., 32, L05109, doi:10.1029/2004GL021445, 2005.

Ukhorskiy, A. Y., Sitnov, M. I., Sharma, A. S., and Papadopoulos, $\mathrm{K}$.: Combining global and multi-scale features in a description of the solar wind-magnetosphere coupling, Ann. Geophys., 21, 1913-1929, 2003.

Uritsky, V. M., Klimas, A. J., and Vassiliadis, D.: Comparative study of dynamical critical scaling in the auroral electrojet index versus solar wind fluctuations, Geophys. Res. Lett., 28(19), 3809-3812, 2001.

Villain, J. P., Caudal, G., and Hanuise, C.: A SAFARI-EISCAT comparison between the velocity of $\mathrm{F}$ region small-scale irregularities and ion drift, J. Geophys. Res., 90, 8433-8444, 1985.

Vörös, Z., Baumjohann, W., Nakamura, R., et al.: Multi-scale magnetic field intermittence in the plasma sheet, Ann. Geophys., 21, 1955-1964, 2003.

Weimer, D. R., Goertz, C. K., Gurnett, D. A., Maynard, N. C., and Burch, J. L.: Auroral zone electric fields from DE 1 and 2 at magnetic conjunctions, J. Geophys. Res., 90, 7479-7494, 1985.

Wild, J. A., Milan, S. E., Owen, C. J., Bosqued, J. M., Lester, M., Wright, D. M., Frey, H., Carlson, C. W., Fazakerley, A. N., and Rème, H.: The location of the open-closed magnetic field line boundary in the dawn sector auroral ionosphere, Ann. Geophys., 22, 3625-3639, 2004

Woodfield, E. E., Davies, J. A., Eglitis, P., and Lester, M.: A case study of HF radar spectral width in the post-midnight magnetic local time sector and its relationship to the polar cap boundary, Ann. Geophys., 20, 501-509, 2002. 